\title{
Points périodiques des fonctions rationnelles dans l'espace hyperbolique $p$-adique
}

\author{
Juan Rivera-Letelier
}

\begin{abstract}
We study the dynamics of rational maps with coefficients in the field $\mathbb{C}_{p}$ acting on the hyperbolic space $\mathbb{H}_{p}$. Our main result is that the number of periodic points in $\mathbb{H}_{p}$ of such a rational map is either 0,1 or $\infty$, and we characterize those rational maps having precisely 0 or 1 periodic points.

The main property we obtain is a criterion for the existence of infinitely many periodic points (of a special kind) in hyperbolic space. The proof of this criterion is analogous to G. Julia's proof of the density of repelling periodic points in the Julia set of a complex rational map.
\end{abstract}

Mathematics Subject Classification (2000). 11S99, 37F10, 51M10, $37 \mathrm{E} 25$.

Keywords. $p$-adic fields, rational maps, hyperbolic space, periodic points.

Soit $p$ un nombre premier. On désigne par $\mathbb{Q}_{p}$ le corps des nombres $p$-adiques et par $\mathbb{C}_{p}$ le complété d'une clôture algébrique de $\mathbb{Q}_{p}$.

Cet article est la suite de [R2 $]^{1}$ dans lequel on a étudié la dynamique des fonctions rationnelles à coefficients dans $\mathbb{C}_{p}$, agissant sur la droite projective $\mathbb{P}\left(\mathbb{C}_{p}\right)$, ainsi que sur l'espace hyperbolique $p$-adique $\mathbb{H}_{p}$.

Cet espace est un arbre réel séparable et complet, isométrique à l'immeuble de Bruhat-Tits de $\operatorname{SL}\left(2, \mathbb{C}_{p}\right)$. De plus, il a un rapport étroit avec l'espace analytique induit par $\mathbb{P}\left(\mathbb{C}_{p}\right)$, au sens de V. G. Berkovich.

Dans ce travail on étudie les points de $\mathbb{H}_{p}$ qui sont périodiques sous l'action d'une fonction rationnelle donnée. Essentiellement, chaque point (rationnel) de $\mathbb{H}_{p}$ qui est fixé par une fonction rationnelle, est en correspondance avec une coordonnée de $\mathbb{P}\left(\mathbb{C}_{p}\right)$ dans laquelle la fonction rationnelle a réduction non triviale. On dit qu'une fonction rationnelle $R$ a réduction non triviale lorsqu'il existe une fonction rationnelle $\widetilde{R}$ à coefficients dans le corps résiduel $\widetilde{\mathbb{C}}_{p}$ de $\mathbb{C}_{p}$ et un sous-ensemble fini $\Xi \operatorname{de} \mathbb{P}\left(\widetilde{\mathbb{C}}_{p}\right)$,

${ }^{1}$ Les résultats de cet article, ainsi que ceux de [R2], ont paru dans le Preprint IMS at Stony Brook \#2001/12. 
tel qu'on ait le diagramme commutatif suivant

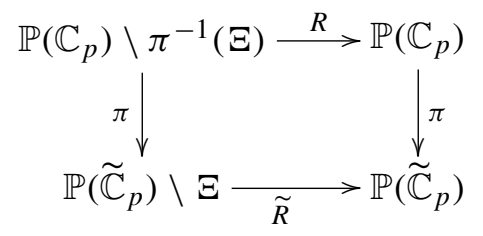

voir [R2]. Ici $\pi$ désigne la projection de $\mathbb{P}\left(\mathbb{C}_{p}\right)$ vers $\mathbb{P}\left(\widetilde{\mathbb{C}}_{p}\right)$.

Le résultat principal qu'on obtient ici est une caractérisation des fonctions rationnelles n'ayant qu'un nombre fini de points périodiques dans $\mathbb{H}_{p}$.

Théorème 1. Le nombre de points périodiques dans $\mathbb{H}_{p}$ d'une fonction rationnelle est égal à 0,1 ou $\infty$.

Autrement dit, pour une fonction rationnelle n'ayant qu'un nombre fini de points périodiques dans $\mathbb{H}_{p}$ il y a deux cas : soit la fonction rationnelle ne possède aucun point périodique dans $\mathbb{H}_{p}$; soit la fonction rationnelle possède un et un seul point périodique dans $\mathbb{H}_{p}$ (dans ce dernier cas le point périodique est un point fixe de la fonction rationnelle).

Dans les théorèmes 2 et 3 ci-dessous on caractérise chacun de ces deux cas. Une fonction rationnelle $R$ a bonne réduction lorsque elle a réduction non triviale et lorsque l'ensemble $\Xi$ ci-dessus est vide, voir $\S 6$ pour une définition plus précise.

Théorème 2. Une fonction rationnelle a un et un seul point périodique dans $\mathbb{H}_{p}$ si et seulement si, après changement de coordonnée, elle a bonne réduction inséparable. Dans ce cas, tous les points périodiques de la fonction rationnelle dans $\mathbb{P}\left(\mathbb{C}_{p}\right)$ sont attractifs.

La notion de bonne réduction d'une fonction rationnelle a été introduite par Morton et Silverman dans [MS], voir aussi [Ben2].

Théorème 3. Pour une fonction rationnelle $R$ de degré au moins 2 à coefficients dans $\mathbb{C}_{p}$, les propriétés suivantes sont équivalentes.

a) $R$ ne possède aucun point périodique dans $\mathbb{H}_{p}$.

b) $R$ possède un seul point périodique non répulsif dans $\mathbb{P}\left(\mathbb{C}_{p}\right)$.

c) $R$ a un nombre fini de points périodiques non répulsifs dans $\mathbb{P}\left(\mathbb{C}_{p}\right)$.

Dans ce cas $R$ a un point fixe attractif dans $\mathbb{P}\left(\mathbb{C}_{p}\right)$ et tous les autres points périodiques de $R$ dans $\mathbb{P}\left(\mathbb{C}_{p}\right)$ sont répulsifs. 
Rappelons que toute fonction rationnelle a un point fixe non répulsif dans $\mathbb{P}\left(\mathbb{C}_{p}\right)$ [Ben1]. Donc, les fonctions rationnelles qui n'ont pas de point périodique dans $\mathbb{H}_{p}$ correspondent au cas extrême où le nombre des points périodiques non répulsifs (comptés avec multiplicité) dans $\mathbb{P}\left(\mathbb{C}_{p}\right)$ est égal à 1 , voir Théorème $\mathrm{A}$ de [R2].

On montrera ailleurs que toute fonction rationnelle dont l'ensemble de Julia est compact et non vide est comme dans l'énoncé du Théorème 3. Ceci généralise aux fonctions rationnelles la Proposition A de [Bez].

Exemple. Fixons un entier $d \geq 2$. Pour $c \in \mathbb{C}_{p}$ on définit $P_{c}(z)=z^{d}+c \in \mathbb{C}_{p}[z]$. Il est facile de voir que si $\left|d^{d} c^{d-1}\right|>1$, alors les points périodiques de $P_{c}$ dans $\mathbb{C}_{p}$ sont répulsifs. Le Théorème 3 implique que le polynôme $P_{c}$ n'a pas de points périodiques dans $\mathbb{H}_{p}$.

D'autre part, dans le cas où $|c| \leq 1$, le polynôme $P_{c}$ a bonne réduction, donnée par le polynôme $\widetilde{P}_{c}(z)=z^{d}+\tilde{c}$. Ainsi, lorsque $p$ divise $d$, le Théorème 2 implique que $P_{c}$ a un et un seul point périodique dans $\mathbb{H}_{p}$.

Le Théorème 3 est une conséquence des résultats établis dans [R2]. Dans la démonstration des théorèmes 1 et 2 , les points exceptionnels dans $\mathbb{H}_{p}$ jouent un rôle essentiel. Un point de $\mathbb{H}_{p}$ est dit exceptionnel lorsque son orbite inverse est finie.

Théorème 4 (Ensemble Exceptionnel). L'ensemble exceptionnel dans $\mathbb{H}_{p}$ contient au plus un point. De plus, il est non vide si et seulement si, après changement de coordonnée, la fonction rationnelle a bonne réduction.

On peut comparer au cas complexe où l'ensemble exceptionnel contient au plus 2 points, cf. [Mi]. Les démonstrations des théorèmes 1 et 2 se déduisent alors du lemme suivant.

Lemme Principal. Soit $R \in \mathbb{C}_{p}(z)$ une fonction rationnelle ayant un point périodique inséparable dans $\mathbb{H}_{p}$ qui n'est pas un point exceptionnel. Alors $R_{*}$ a une infinité de points périodiques inséparables dans $\mathbb{H}_{p}$.

La démonstration du lemme est analogue à la démonstration de G. Julia concernant la densité des points périodiques sur l'ensemble qui porte son nom (voir [Mi]).

D'une façon surprenante, un raisonnement assez proche de la démonstration du Théorème 4 permet de donner une preuve « conceptuelle » du théorème suivant, dû à R. Benedetto.

Théorème ([Ben2], Theorem $\mathrm{B})$. Si $R \in \mathbb{C}_{p}(z)$ est une fonction rationnelle de degré au moins deux et si $n$ est un entier positif, alors $R^{n}$ a bonne réduction si et seulement si $R$ a bonne réduction. 
Plan de l'article. Dans les paragraphes 1, 2 et 3 on rappelle quelque définitions et résultats concernant le corps $\mathbb{C}_{p}$ et l'espace $\mathbb{H}_{p}$. Dans les paragraphes 4 et 5 on étudie les points périodiques inséparables. Dans les paragraphes 6 et 7 on étudie l'ensemble exceptionnel dans $\mathbb{H}_{p}$, et on donne la démonstration du Théorème 4 . Dans l'appendice (paragraphe 12) on établit une propriété générale qui est nécessaire pour la démonstration du Théorème 4 .

Dans le paragraphe 8 on obtient les théorèmes 1 et 2 à partir du Lemme Principal. On montre aussi le Théorème 3 avec les résultats de [R2]. Les paragraphes 9, 10 et 11 sont consacrés à la démonstration du Lemme Principal.

Remerciements. Je remercie J. C. Yoccoz et R. Benedetto pour plusieurs remarques et corrections qu'ils ont faites concernant une version préliminaire de ce travail. Je remercie aussi le Collège de France pour son hospitalité. Je remercie le rapporteur dont ses remarques et corrections ont beaucoup aidées à améliorer l'exposition de cet article.

\section{Préliminaires}

Soit $p>1$ un nombre premier, $\mathbb{Q}_{p}$ le corps des nombres $p$-adiques et $\mathbb{C}_{p}$ le complété d'une clôture algébrique de $\mathbb{Q}_{p}$.

On désigne par $|\cdot|$ la norme sur $\mathbb{C}_{p}$ et $\mathbb{C}_{p}^{*}=\mathbb{C}_{p} \backslash\{0\}$ le groupe multiplicatif de $\mathbb{C}_{p}$. On appelle

$$
\begin{aligned}
\left|\mathbb{C}_{p}^{*}\right| & =\left\{|z| \mid z \in \mathbb{C}_{p}^{*}\right\} \\
& =\left\{r>0 \mid \log _{p} r \text { est rationnel }\right\}
\end{aligned}
$$

le groupe de valuation de $\mathbb{C}_{p}^{*}$.

On désigne par $\mathcal{O}_{p}=\left\{z \in \mathbb{C}_{p}|| z \mid \leq 1\right\}$ l'anneau des entiers. L'ensemble $\mathfrak{m}_{p}=\left\{z \in \mathbb{C}_{p}|| z \mid<1\right\}$ est un idéal maximal de $\mathcal{O}_{p}$. Le corps $\widetilde{\mathbb{C}}_{p}=\mathcal{O}_{p} / \mathfrak{m}_{p}$ est appelé le corps résiduel de $\mathbb{C}_{p}$. Il est isomorphe à une clôture algébrique $\overline{\mathbb{F}}_{p}$ du corps fini $\mathbb{F}_{p}$. On identifie $\widetilde{\mathbb{C}}_{p}$ à $\overline{\mathbb{F}}_{p}$.

Pour $z \in \mathcal{O}_{p}$ on désigne par $\tilde{z}$ la projection de $z$ dans $\overline{\mathbb{F}}_{p}$. Pour $\zeta \in \overline{\mathbb{F}}_{p}$ on pose $B(\zeta)=\{\tilde{z}=\zeta\}$, de sorte qu'on a la partition,

$$
\mathcal{O}_{p}=\sqcup_{\overline{\mathbb{F}}_{p}} B(\zeta)
$$

1.1. La droite projective. On considère la droite projective $\mathbb{P}\left(\mathbb{C}_{p}\right)$, qui est l'ensemble des droites dans $\mathbb{C}_{p} \times \mathbb{C}_{p}$ passant par $(0,0)$. Pour $(x, y) \in \mathbb{C}_{p} \times \mathbb{C}_{p} \backslash\{(0,0)\}$, on désigne par $[x, y] \in \mathbb{P}\left(\mathbb{C}_{p}\right)$ le point correspondant à la droite $\left\{(\lambda x, \lambda y) \mid \lambda \in \mathbb{C}_{p}\right\}$. On désigne par $\infty$ le point $[1,0] \in \mathbb{P}\left(\mathbb{C}_{p}\right)$, et on identifie $\mathbb{P}\left(\mathbb{C}_{p}\right) \backslash\{\infty\}$ à $\mathbb{C}_{p}$ par l'application $[\lambda, 1] \mapsto \lambda$. 
On étend la projection de $\mathbb{C}_{p}$ vers $\overline{\mathbb{F}}_{p}$ en une projection de $\mathbb{P}\left(\mathbb{C}_{p}\right)=\mathbb{C}_{p} \cup\{\infty\}$ vers $\mathbb{P}\left(\overline{\mathbb{F}}_{p}\right)=\overline{\mathbb{F}}_{p} \cup\{\infty\}$, par $\tilde{z}=\infty$, pour $z \in\{|z|>1\} \cup\{\infty\}$. On pose $B(\infty)=$ $\{\tilde{z}=\infty\}=\{|z|>1\} \cup\{\infty\}$. On a alors la partition canonique

$$
\mathbb{P}\left(\mathbb{C}_{p}\right)=\sqcup_{\mathbb{P}\left(\overline{\mathbb{F}}_{p}\right)} B(\zeta) .
$$

Pour chaque $a, b, c, d \in \mathbb{C}_{p}$ tel que $a d-b c \neq 0$ l'application linéaire $(x, y) \mapsto$ $(a x+b y, c x+d y)$ de $\mathbb{C}_{p} \times \mathbb{C}_{p}$ dans lui même induit une application de $\mathbb{P}\left(\mathbb{C}_{p}\right)$ dans lui même, qu'on appelle transformation projective. Les transformations projectives forment un groupe isomorphe à $\operatorname{PGL}\left(2, \mathbb{C}_{p}\right):$ l'élément $\left(\begin{array}{ll}a & c \\ b & d\end{array}\right) \in \operatorname{PGL}\left(2, \mathbb{C}_{p}\right)$ correspond à la transformation projective $\operatorname{de} \mathbb{P}\left(\mathbb{C}_{p}\right)$ induite par $(x, y) \mapsto(a x+b y, c x+d y)$.

Le sous-groupe $\operatorname{PGL}\left(2, \mathcal{O}_{p}\right)$ de $\operatorname{PGL}\left(2, \mathbb{C}_{p}\right)$ correspond à celui constitué des transformations projectives qui préservent la partition (1). De plus, la transformation projective de $\mathbb{P}\left(\mathbb{C}_{p}\right)$ associé à $\left(\begin{array}{ll}a & b \\ c & d\end{array}\right) \in \operatorname{PGL}\left(2, \mathcal{O}_{p}\right)$ préserve chaque élément de la partition (1) si et seulement si $a, d \in 1+\mathfrak{m}_{p}$ et $b, c \in \mathfrak{m}_{p}$.

1.2. Boules et couronnes. Étant donnés $r \in\left|\mathbb{C}_{p}^{*}\right|$ et $a \in \mathbb{C}_{p}$, on appelle les ensembles

$$
\left\{z \in \mathbb{C}_{p}|| z-a \mid<r\right\} \quad \text { et } \quad\left\{z \in \mathbb{C}_{p}|| z-a \mid \leq r\right\}
$$

boule ouverte de $\mathbb{C}_{p}$ et boule fermée de $\mathbb{C}_{p}$, respectivement. Si $r \notin\left|\mathbb{C}_{p}^{*}\right|$ alors ces deux ensembles coïncident et constituent ce qu' on appelle une boule irrationnelle de $\mathbb{C}_{p}$. Notons que par définition une boule $B$ de $\mathbb{C}_{p}$ est irrationnelle si et seulement si $\operatorname{diam}(B) \notin\left|\mathbb{C}_{p}^{*}\right|$; en particulier, si $B$ est ouverte ou fermée alors $\operatorname{diam}(B) \in\left|\mathbb{C}_{p}^{*}\right|$.

Étant donnés deux boules $B$ et $B^{\prime}$ de $\mathbb{C}_{p}$ ayant une intersection non vide, il y a deux possibilités : soit $B \subset B^{\prime}$, soit $B^{\prime} \subset B$.

L'image d'une boule ouverte (resp. fermée, irrationnelle) par une transformation affine de $\mathbb{C}_{p}$ est une boule de même nature.

Une boule ouverte (resp. fermée, irrationnelle) de $\mathbb{P}\left(\mathbb{C}_{p}\right)$ est soit une boule de $\mathbb{C}_{p}$ de même nature, soit le complémentaire d'une boule fermée (resp. ouverte, resp. irrationnelle) de $\mathbb{C}_{p}$. Dans ce qui suit le mot boule désignera une boule de $\mathbb{P}\left(\mathbb{C}_{p}\right)$.

Étant données deux boules $B$ et $B^{\prime}$ de $\mathbb{P}\left(\mathbb{C}_{p}\right)$ qui s'intersectent, il y a trois possibilités : soit $B \subset B^{\prime}$, soit $B^{\prime} \subset B$, soit $B \cup B^{\prime}=\mathbb{P}\left(\mathbb{C}_{p}\right)$. Dans ce dernier cas les complémentaires de $B$ et $B^{\prime}$ sont disjoints; si de plus $B$ et $B^{\prime}$ ne sont pas fermées alors on dit que $B \cap B^{\prime}$ est une couronne. Après changement de coordonnée, on peut supposer $B=\{|z|<r\}$ et $B^{\prime}=\left\{|z|>r^{\prime}\right\} \cup\{\infty\}$ avec $r^{\prime}<r$; alors

$$
B \cap B^{\prime}=\left\{z \in \mathbb{C}_{p}\left|r^{\prime}<\right| z \mid<r\right\} .
$$

On définit $\bmod \left(B \cap B^{\prime}\right)=\log _{p} r-\log _{p} r^{\prime}>0$. La valeur de cette expression ne dépend pas du choix de coordonnée, et on l'appelle le module de la couronne $B \cap B^{\prime}$.

L'image d'une boule ouverte (resp. fermée, irrationnelle) par une transformation projective de $\mathbb{P}\left(\mathbb{C}_{p}\right)$ est une boule de même nature. 
1.3. Séries convergentes. Dans ce paragraphe on considère quelques résultats basiques d'analyse ultramétrique. Pour les démonstrations le lecteur pourra consulter, par exemple, [BGR].

Une série $f(z)=a_{0}+a_{1} z+\cdots$ à coefficients dans $\mathbb{C}_{p}$ converge sur la boule $\{|z|<r\}$ si et seulement si $\lim \sup _{i \rightarrow \infty}\left|a_{i}\right|^{1 / i} \leq r^{-1}$. Lorsque $r \in\left|\mathbb{C}_{p}^{*}\right|$ la série $f$ converge sur la boule fermée $\{|z| \leq r\}$ si et seulement si $\lim _{\sup _{i \rightarrow \infty}}\left|a_{i}\right| r^{i}<\infty$. Dans ces deux cas pour tout $r_{0} \in(0, r]$ on a

$$
\|f\|_{r_{0}}:=\sup \left\{|f(z)||| z \mid<r_{0}\right\}=\sup \left\{\left|a_{i}\right| r_{0}^{i} \mid i \geq 0\right\} .
$$

Lorsque $f$ converge sur $\left\{|z| \leq r_{0}\right\}$ on a aussi $\|f\|_{r_{0}}=\sup \left\{|f(z)||| z \mid \leq r_{0}\right\}$. De plus, $f$ est injective sur la boule $\{|z|<r\}$ si et seulement si $a_{1} \neq 0$ et pour tout $i>1$ on a $\left|a_{i}\right| r^{i-1} \leq\left|a_{1}\right|$. Dans ce cas l'image de $\{|z|<r\}$ par $f$ est égal à la boule $\left\{\left|z-a_{0}\right|<\left|a_{1}\right| r\right\}$.

Toute fonction rationnelle à coefficients dans $\mathbb{C}_{p}$ admet un développement en série en chaque point $z_{0}$ de $\mathbb{C}_{p}$ qui n'est pas un pôle. Le rayon de convergence est égal à la plus petite distance entre $z_{0}$ et un pôle.

Si $f$ est une série convergente sur une boule $B$ de $\mathbb{C}_{p}$, alors l'image par $f$ d'une boule ouverte (resp. irrationnelle, fermée) strictement contenue dans $B$ est une boule de même nature.

Lemme 1.1. Soient $f$ et $\varepsilon$ des séries convergentes sur une boule $B$ de $\mathbb{C}_{p}$. Si D est une boule strictement contenue dans $B$ et $\sup \{|\varepsilon(z)| \mid z \in D\}<\operatorname{diam}(f(D))$, alors $(f+\varepsilon)(D)=f(D)$.

Preuve. On sait que $(f+\varepsilon)(D)$ et $f(D)$ sont des boules de même nature. L'hypothèse implique que $(f+\varepsilon)(D) \subset f(D)$ et que $\operatorname{diam}((f+\varepsilon)(D))=\operatorname{diam}(f(D))$. On a donc $(f+\varepsilon)(D)=f(D)$.

Lemme 1.2. Soient $r, r^{\prime}>0$ et considérons une série $f$ convergente sur $\{|z|<r\}$, telle que $f(\{|z|<r\}) \subset\left\{|z|<r^{\prime}\right\}$. Alors pour toute boule $D \subset\{|z|<r\}$ on a

$$
\frac{1}{r^{\prime}} \operatorname{diam}(f(D)) \leq \frac{1}{r} \operatorname{diam}(D) .
$$

Si de plus $\operatorname{diam}(D)<r$, alors on a l'égalité si et seulement si $f$ induit une bijection entre $\{|z|<r\}$ et $\left\{|z|<r^{\prime}\right\}$.

Preuve. Soit $a \in D$ et posons $g(z)=f(z+a)=b_{0}+b_{1} z+\cdots$. Par hypothèse on a

$$
\|f\|_{r}=\|g\|_{r}=\sup \left\{\left|b_{i}\right| r^{i} \mid i \geq 0\right\} \leq r^{\prime} .
$$

Si l'on pose $r_{0}=\operatorname{diam}(D)$, alors on a

$$
\operatorname{diam}(f(D))=\left\|g-b_{0}\right\|_{r_{0}}=\sup \left\{\left|b_{i}\right| r_{0}^{i} \mid i \geq 1\right\} .
$$


Mais pour chaque entier $i \geq 1$ on a $\left|b_{i}\right| r_{0}^{i} \leq r^{\prime}\left(r_{0} / r\right)^{i} \leq r^{\prime}\left(r_{0} / r\right)$, d'où on obtient l'inégalité désirée. Lorsque $r_{0}<r$, on a l'égalité si et seulement si $\left|b_{1}\right|=r^{\prime} / r$. Mais $g$ (et donc $f$ ) induit une bijection entre $\{|z|<r\}$ et $\left\{|z|<r^{\prime}\right\}$ si et seulement si $\left|b_{1}\right|=r^{\prime} / r$.

\section{Espace hyperbolique $\mathbb{H}_{p}$}

Dans ce paragraphe on fait des rappels sur l'espace hyperbolique $\mathbb{H}_{p}$. On trouvera les détails dans les paragraphes 3 et 4 de [R2].

2.1. Bouts. Soit $\left\{B_{i}\right\}_{i \geq 0}$ une suite croissante de boules fermées ou irrationnelles telle que $B=\bigcup_{i \geq 0} B_{i}$ soit une boule ouverte ou irrationnelle, ou soit égale à $\mathbb{P}\left(\mathbb{C}_{p}\right)$. Alors $\left\{B \backslash B_{i}\right\}_{i \geq 0}$ est soit une suite décroissante de couronnes, soit une suite décroissante de boules, respectivement. On appelle $\left\{B \backslash B_{i}\right\}_{i \geq 0}$ chaîne évanescente. Notons qu'on a

$$
\bigcap_{i \geq 0}\left(B \backslash B_{i}\right)=\varnothing
$$

et par conséquent $B \backslash B_{i} \subset \mathbb{C}_{p}$, pour $i$ assez grand. De plus, $\operatorname{diam}\left(B \backslash B_{i}\right)$ converge vers un nombre positif lorsque $i \rightarrow \infty$.

On dit que deux chaînes évanescentes $\left\{B \backslash B_{i}\right\}_{i \geq 0}$ et $\left\{B^{\prime} \backslash B_{i}^{\prime}\right\}_{i \geq 0}$ sont équivalentes si pour tout $N \geq 0$ il existe $n \geq N$ tel que $B_{N} \subset B_{n}^{\prime}$ et $B_{N}^{\prime} \subset B_{n}$. Dans ce cas $B=B^{\prime}$.

Définition 2.1. Un bout est une classe d'équivalence de chaînes évanescentes.

Soit $\mathcal{P}$ un bout et $\left\{B \backslash B_{i}\right\}_{i \geq 0}$ une chaîne évanescente définissante. Alors $B$ dépend seulement de $\mathcal{P}$ et on pose $B_{\mathcal{P}}=B$.

Si $B_{\mathcal{P}}=\mathbb{P}\left(\mathbb{C}_{p}\right)$, alors on dit que $\mathcal{P}$ est un bout singulier. Sinon $B_{\mathcal{P}}$ est une boule ouverte ou irrationnelle qui est déterminée par $\mathcal{P}$. Si $B_{\mathcal{P}}$ est une boule ouverte (resp. irrationnelle) alors on dit que $\mathcal{P}$ est rationnel (resp. irrationnel). On a une correspondance entre les boules ouvertes (resp. irrationnelles) et les bouts rationnels (resp. irrationnels)

Chaque transformation projective $\varphi$ de $\mathbb{P}\left(\mathbb{C}_{p}\right)$ induit une bijection sur les bouts rationnels (resp. irrationnels, singuliers). On désigne cette action par $\varphi_{*}$.

2.2. Partitions de la droite projective et points de $\mathbb{H}_{p}$. L'espace hyperbolique, qu'on désigne par $\mathbb{H}_{p}$, est par définition un ensemble de points, qu'on décrit cidessous. Il y a trois types de points de $\mathbb{H}_{p}$ : les points singuliers, rationnels et irrationnels.

2.2.1. Points singuliers. Les points singuliers de $\mathbb{H}_{p}$ sont par définition les ensembles de la forme $\delta=\{\mathcal{P}\}$, où $\mathcal{P}$ est un bout singulier. 
2.2.2. Points non singuliers. On dit que deux boules ouvertes ou irrationnelles $B_{0}$ et $B_{1}$ sont associées, si $B_{0} \cap B_{1}=\varnothing$ et si $B_{0}$ et $B_{1}$ sont maximales pour cette propriété. Autrement dit, si $i \in\{0,1\}$ et $B_{i}^{\prime}$ est une boule ouverte ou irrationnelle telle que $B_{i} \subset B_{i}^{\prime}$ et $B_{i}^{\prime} \cap B_{1-i}=\emptyset$, alors $B_{i}^{\prime}=B_{i}$.

Lemme 2.2. Soient $B_{0}$ et $B_{1}$ associées à B. Alors $B_{0}=B_{1}$ ou $B_{0} \cap B_{1}=\emptyset$. Dans ce dernier cas $B_{0}$ est associée à $B_{1}$.

Preuve. La première assertion suit par maximalité. Supposons $B_{0} \cap B_{1}=\emptyset$. Soit $i \in$ $\{0,1\}$ et soit $B_{i}^{\prime}$ une boule ouverte ou irrationnelle telle que $B_{i} \subset B_{i}^{\prime}$ et $B_{i}^{\prime} \cap B_{1-i}=\emptyset$. Alors $B \not \subset B_{i}^{\prime}$, car $B_{1-i}$ est associée à $B$, donc $B_{i}^{\prime} \cap B=\emptyset$. Par conséquent $B_{i}^{\prime}=B_{i}$, car $B_{i}$ est associée à $B$.

Un point non singulier $\delta$ de $\mathbb{H}_{p}$ est par définition un ensemble de bouts rationnels ou irrationnels tel que pour tous $\mathcal{P}_{0}$ et $\mathcal{P}_{1} \in \&$ distincts, les boules $B_{\mathcal{P}_{0}}$ et $B_{\mathcal{P}_{1}}$ soient associées, et maximales pour cette propriété. Dans ce cas on dit qu'une boule $B_{\mathcal{P}}$, avec $\mathcal{P} \in \&$, est associée à $\&$.

Notons que l'union d'une suite croissante de boules ouvertes ou irrationnelles disjointes d'une boule donnée, est une boule ouverte ou irrationnelle. Par conséquent chaque point non singulier $\&$ de $\mathbb{H}_{p}$ contient au moins deux éléments, et on a la partition

$$
\mathbb{P}\left(\mathbb{C}_{p}\right)=\sqcup_{\S} B_{\mathcal{P}} .
$$

2.2.3. Points irrationnels. Étant donné un bout irrationnel $\mathcal{P}$, les ensembles $B_{\mathcal{P}}$ et $\mathbb{P}\left(\mathbb{C}_{p}\right) \backslash B_{\mathcal{P}}$ sont des boules irrationnelles. Alors $\left\{\mathcal{P}, \mathcal{P}^{\prime}\right\}$ est un point non singulier de $\mathbb{H}_{p}$, où $\mathcal{P}^{\prime}$ est le bout associé à $\mathbb{P}\left(\mathbb{C}_{p}\right) \backslash B_{\mathcal{P}}$. On appelle $\left\{\mathcal{P}, \mathcal{P}^{\prime}\right\}$ point irrationnel.

2.2.4. Le point canonique. Rappelons que pour $\zeta \in \mathbb{P}\left(\overline{\mathbb{F}}_{p}\right)$ on désigne par $B(\zeta)$ la boule $\left\{z \in \mathbb{P}\left(\mathbb{C}_{p}\right) \mid \tilde{z}=\zeta\right\}$; voir Préliminaires. On a la partition canonique

$$
\mathbb{P}\left(\mathbb{C}_{p}\right)=\sqcup_{\mathbb{P}\left(\overline{\mathbb{F}}_{p}\right)} B(\zeta)
$$

Soit $\mathcal{P}(\zeta)$ le bout correspondant à $B(\zeta)$. Il est facile de voir que $\varsigma_{\text {can }}=\{\mathcal{P}(\zeta)\}_{\mathbb{P}\left(\overline{\mathbb{F}}_{p}\right)}$ est un point non singulier. On l'appelle le point canonique.

2.2.5. Points rationnels. Soit $\mathcal{P}$ un bout rationnel et soit $\varphi$ une transformation projective de $\mathbb{P}\left(\mathbb{C}_{p}\right)$ tel que $\varphi(\{|z|<1\})=B_{\mathcal{P}}$. Alors $\mathcal{P}=\varphi_{*}(\mathcal{P}(0))$ et $\delta=$ $\left\{\varphi_{*}(\mathcal{P}(\zeta))\right\}_{\mathbb{P}\left(\overline{\mathbb{F}}_{p}\right)}$ est un point non singulier contenant $\mathcal{P}$. On appelle $s \in \mathbb{H}_{p}$ point rationnel. En particulier, $\wp_{\text {can }}$ est un point rationnel.

Notons qu'on a un paramétrage de $\&$ par $\mathbb{P}\left(\overline{\mathbb{F}}_{p}\right)$ qui est unique, modulo un changement de coordonnée projective de $\mathbb{P}\left(\overline{\mathbb{F}}_{p}\right)$. 


\subsubsection{Définition de l'espace hyperbolique}

Définition 2.3. L'espace hyperbolique p-adique, qu'on désigne par $\mathbb{H}_{p}$, est l'ensemble des points rationnels, irrationnels et singuliers. De plus, on désigne par $\mathbb{H}_{p}^{\mathbb{R}}$ (resp. $\mathbb{H}_{p}^{\mathbb{Q}}$ ) l'ensemble des points non singuliers (resp. rationnels) de $\mathbb{H}_{p}$.

Notons que tout bout (resp. bout non singulier, bout rationnel) est contenu dans exactement un point de $\mathbb{H}_{p}$ (resp. $\mathbb{H}_{p}^{\mathbb{R}}, \mathbb{H}_{p}^{\mathbb{Q}}$ ).

Il est clair que le groupe $\operatorname{PGL}\left(2, \mathbb{C}_{p}\right)$ des transformations projectives de $\mathbb{P}\left(\mathbb{C}_{p}\right)$ agit $\operatorname{sur} \mathbb{H}_{p}$, en préservant $\mathbb{H}_{p}^{\mathbb{R}}$ et $\mathbb{H}_{p}^{\mathbb{Q}}$. Cette action est transitive sur $\mathbb{H}_{p}^{\mathbb{Q}}$ et le stabilisateur du point $\oint_{\text {can }}$ correspond au sous-groupe PGL $\left(2, \mathcal{O}_{p}\right)$. Par conséquent on a une bijection entre $\operatorname{PGL}\left(2, \mathbb{C}_{p}\right) / \operatorname{PGL}\left(2, \mathcal{O}_{p}\right)$ et $\mathbb{H}_{p}^{\mathbb{Q}}$. Étant donné une transformation projective $\varphi$ de $\mathbb{P}\left(\mathbb{C}_{p}\right)$, on désigne par $\varphi_{*}$ l'action sur $\mathbb{H}_{p}$ induite par $\varphi$.

\subsection{Propriété de séparation}

Définition 2.4. Soit $\delta \in \mathbb{H}_{p}$ et $X \subset \mathbb{P}\left(\mathbb{C}_{p}\right)$.

1. Si $\& \in \mathbb{H}_{p}^{\mathbb{R}}$ est non singulier et si $X$ intersecte au moins deux boules associées à $\delta$, alors on dit que $\&$ sépare $X$, et on note $\delta \prec X$.

2. Si $\delta=\{\mathcal{P}\} \in \mathbb{H}_{p}$ est singulier et si pour toute chaîne évanescente $\left\{D_{i}\right\}_{i \geq 0}$ définissant $\mathcal{P}$ et tout $i \geq 0$ on a $D_{i} \cap X \neq \emptyset$, alors on écrit $\delta \prec X$.

Soient $s \in \mathbb{H}_{p}$ et $X, Y \subset \mathbb{P}\left(\mathbb{C}_{p}\right)$. Alors $(s \prec X$ et $X \subset Y$ ) implique $s \prec Y$. Notons que pour un point singulier $\delta=\{\mathcal{P}\}$ il suffit de vérifier la propriété 2 pour une chaîne évanescente définissant $\mathcal{P}$ quelconque.

Lemme 2.5. Soient $\&$ et $\S^{\prime} \in \mathbb{H}_{p}$ des points distincts. Alors il existe un unique bout $\mathcal{P} \in \&$ tel que $\S^{\prime} \prec B_{\mathcal{P}}$.

Lemme 2.6. Soient $\&, \delta^{\prime} \in \mathbb{H}_{p}^{\mathbb{R}}$ distincts. Soit $B$ (resp. $B^{\prime}$ ) la boule associée à $s$ (resp. $\delta^{\prime}$ ) telle que $\delta^{\prime} \prec B$ (resp. $\& \prec B^{\prime}$ ). Alors $B \cap B^{\prime}$ est une couronne.

2.4. Propriété de séparation dans $\mathbb{H}_{p}$. Fixons un point $\delta \in \mathbb{H}_{p}$. Par le Lemme 2.5, chaque point $\delta^{\prime} \in \mathbb{H}_{p}$ différent de $\delta$ détermine un bout $\mathcal{P} \in \delta$ tel que $\delta^{\prime} \prec B_{\mathcal{P}}$.

Comme $\mathbb{P}\left(\mathbb{C}_{p}\right)=\sqcup_{\S} B_{\mathcal{P}}$ est une partition de $\mathbb{P}\left(\mathbb{C}_{p}\right)$, chaque point $z^{\prime} \in \mathbb{P}\left(\mathbb{C}_{p}\right)$ détermine un bout $\mathcal{P} \in \mathcal{\&}$ tel que $z^{\prime} \in B_{\mathcal{P}}$. On écrit $z^{\prime} \prec B_{\mathcal{P}}$.

Définition 2.7. Soient $\S_{0}, \delta_{1} \in \mathbb{H}_{p} \cup \mathbb{P}\left(\mathbb{C}_{p}\right)$ et $\delta \in \mathbb{H}_{p}$ des points deux à deux distincts. Pour $i \in\{0,1\}$ soit $\mathcal{P}_{i} \in \&$ le bout tel que $\delta_{i} \prec B_{\mathcal{P}_{i}}$. On dit que $\&$ est entre $\delta_{0}$ et $\delta_{1}$ si $\mathcal{P}_{0} \neq \mathcal{P}_{1}$. On dit aussi que $\delta$ sépare $\varsigma_{0}$ et $\S_{1}$.

On désigne par $\left(\wp_{0}, \wp_{1}\right) \subset \mathbb{H}_{p}$ l'ensemble de tous les points entre $\varsigma_{0}$ et $\varsigma_{1}$. De

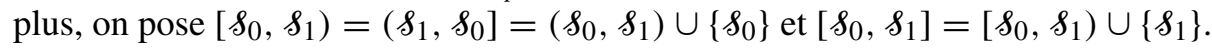


Notons qu'un point $\delta \in \mathbb{H}_{p}$ peut séparer deux éléments de $\mathbb{H}_{p} \cup \mathbb{P}\left(\mathbb{C}_{p}\right)$ si et seulement si $\delta \in \mathbb{H}_{p}^{\mathbb{R}}$. Cette définition généralise la définition 2.4 dans la mesure où un point $\delta \in \mathbb{H}_{p}^{\mathbb{R}}$ sépare deux points $z_{0}$ et $z_{1} \in \mathbb{P}\left(\mathbb{C}_{p}\right)$ distincts si et seulement si $\&$ sépare l'ensemble $\left\{z_{0}, z_{1}\right\} \subset \mathbb{P}\left(\mathbb{C}_{p}\right)$ (dans le sens de la définition 2.4).

Lemme 2.8. Soient $\varsigma_{0}, \varsigma_{1}$ et $\varsigma_{2} \in \mathbb{H}_{p} \cup \mathbb{P}\left(\mathbb{C}_{p}\right)$ des points distincts tels que $\varsigma_{i}$ ne soit pas entre $\delta_{j}$ et $\delta_{k}$, pour toutes les choix de $\{i, j, k\}=\{0,1,2\}$. Alors il existe un unique point $s \in \mathbb{H}_{p}$ tel que $s$ est entre $\varsigma_{0}$ et $\delta_{1}$, entre $\varsigma_{1}$ et $\delta_{2}$ et entre $\varsigma_{2}$ et $\varsigma_{0}$. Dans ce cas $\& \in \mathbb{H}_{p}^{\mathbb{Q}}$.

Lemme 2.9. Soit $\mathcal{P}$ un bout et soient $\S_{0}, \S_{1} \in \mathbb{H}_{p}$ des points distincts tels que $\varsigma_{0}, \varsigma_{1} \prec B_{\mathcal{P}}$. Alors il existe un point $\bar{s} \in \mathbb{H}_{p}^{\mathbb{R}}$ tel que $\bar{s} \prec B_{\mathcal{P}}$ et tel que

$$
\left[\S_{0}, \S\right) \cap\left[\wp_{1}, \S\right)=[\bar{\S}, \S)
$$

où s est le point de $\mathbb{H}_{p}$ contenant $\mathcal{P}$.

2.5. Distance sur $\mathbb{H}_{p}$. L'espace hyperbolique $\mathbb{H}_{p}$ est muni d'une distance $d$, pour laquelle il est un arbre réel séparable et complet, voir le paragraphe 3 de [R2]. De plus, cette distance est invariante par l'action des homographies sur $\mathbb{H}_{p}$.

Pour des points non singuliers distincts $\delta$ et $\delta^{\prime}$ de $\mathbb{H}_{p}^{\mathbb{R}}$, cette distance est définie comme suit. Soit $B$ la boule associée à $\delta$ telle que $\delta^{\prime} \prec B$ et soit $B^{\prime}$ la boule associée à $\delta^{\prime}$ telle que $s \prec B^{\prime}$ (voir Lemme 2.5). Par le Lemme 2.6, $B \cap B^{\prime}$ est une couronne et alors

$$
d\left(\delta, f^{\prime}\right)=\bmod \left(B \cap B^{\prime}\right) .
$$

Il est facile de voir que si $D$ (resp. $D^{\prime}$ ) est une boule de $\mathbb{C}_{p}$ associée à $\&$ (resp. $\mathcal{\delta}^{\prime}$ ) on a

$$
d\left(\delta, f^{\prime}\right)=\log _{p} \frac{\operatorname{diam}\left(D \cup D^{\prime}\right)^{2}}{\operatorname{diam}(D) \cdot \operatorname{diam}\left(D^{\prime}\right)} .
$$

Les segments géodésiques de l'arbre réel $\left(\mathbb{H}_{p}, d\right)$ sont les ensembles de la forme $\left[\&, 8^{\prime}\right]$, voir Définition 2.7. On sait que les points rationnels sont denses sur chaque segment géodésique.

Pour $z, z^{\prime} \in \mathbb{P}\left(\mathbb{C}_{p}\right)$ on dit que $\left(z, z^{\prime}\right) \subset \mathbb{H}_{p}^{\mathbb{R}}$ est la géodésique joignant $z$ et $z^{\prime}$. Chaque géodesique est isométrique à $\mathbb{R}$. Dans le cas où $\& \in \mathbb{H}_{p}$ et $z \in \mathbb{P}\left(\mathbb{C}_{p}\right)$, on dit que $[\delta, z)$ est une demi-géodésique. Si $\&$ appartient à $\mathbb{H}_{p}^{\mathbb{R}}$ alors il existe une transformation projective de $\mathbb{P}\left(\mathbb{C}_{p}\right)$ envoyant $z$ à $\infty$ et $\delta$ dans $(0, \infty)$. Par conséquent $[\delta, z)$ est isométrique à $[0, \infty) \subset \mathbb{R}$.

Lorsqu'on fixe $\delta \in \mathbb{H}_{p}$ et $\delta^{\prime}$ se rapproche (sur une géodésique) d'un point de $\mathbb{P}\left(\mathbb{C}_{p}\right)$, alors la distance $d\left(\delta, \delta^{\prime}\right)$ tend vers l'infini. 
2.6. Remarques à propos de l'espace de Berkovich. Comme ensemble, l'espace analytique de Berkovich $\mathbb{P}_{\mathbb{C}_{p}}^{\text {an }}$ induit par $\mathbb{P}\left(\mathbb{C}_{p}\right)$ s'identifie de façon naturelle à l'ensemble $\mathbb{P}\left(\mathbb{C}_{p}\right) \sqcup \mathbb{H}_{p}$. Les points de $\mathbb{P}\left(\mathbb{C}_{p}\right)$ s'identifient aux points de type (i) de $\mathbb{P}_{\mathbb{C}_{p}}^{\text {an }}$ et les points rationnels (resp. irrationnels, singuliers) de $\mathbb{H}_{p}$ s'identifient aux points de type (ii) (resp. (iii), (iv)) de $\mathbb{P}_{\mathbb{C}_{p}}^{\text {an }}$.

L'espace $\mathbb{P}_{\mathbb{C}_{p}}^{\text {an }}$ est munit d'une topologie pour laquelle il est compact et connexe par arcs. La topologie sur $\mathbb{H}_{p}$ induite par $\mathbb{P}_{\mathbb{C}_{p}}^{\text {an }}$ est strictement moins fine que celle induite par la distance $d$.

Étant donné un point $s$ de $\mathbb{P}\left(\mathbb{C}_{p}\right) \sqcup \mathbb{H}_{p}=\mathbb{P}_{\mathbb{C}_{p}}^{\text {an }}$ et une boule $B$ de $\mathbb{P}\left(\mathbb{C}_{p}\right)$, on a $\delta \prec B$ si et seulement si $\delta \in B^{\text {an }}$, où $B^{\text {an }}$ désigne l'espace analytique de Berkovich induit par $B$. De plus, pour chaque point $\delta \in \mathbb{H}_{p}$ on a la partition

$$
\mathbb{P}_{\mathbb{C}_{p}}^{\text {an }} \backslash\{\delta\}=\sqcup \sqcup_{\mathcal{P} \in \mathcal{S}} B_{\mathcal{P}}^{\text {an }}
$$

(Lemme 2.5), laquelle est la partition en composantes connexes de $\mathbb{P}_{\mathbb{C}_{p}}^{\text {an }} \backslash\{\S\}$.

\section{Action des fonctions rationnelles sur $\mathbb{H}_{p}$}

Fixons une fonction rationnelle $R \in \mathbb{C}_{p}(z)$ qui ne soit pas constante. Dans ce paragraphe on décrit l'action d'une fonction rationnelle sur les bouts et sur $\mathbb{H}_{p} ;$ pour les démonstrations on pourra consulter le paragraphe 4 de [R2].

Étant donné un point $w \in \mathbb{P}\left(\mathbb{C}_{p}\right)$, le degré local de $R$ en $w$, que l'on désigne par $\operatorname{deg}_{R}(w)$, est défini comme suit. On considère des coordonnées telles que $w=0$ et $R(0)=0$. Alors $R$ est localement de la forme

$$
a_{d} z^{d}+a_{d+1} z^{d+1}+\cdots, \quad \text { où } d \geq 1 \text { et } a_{d} \neq 0 ;
$$

on définit $\operatorname{deg}_{R}(w)=d$ et on dit que $\operatorname{deg}_{R}(w)$ est la multiplicité de $w$ comme antécédent de $R(w)$. Il n'est pas difficile de voir que $\operatorname{deg}_{R}(w)$ ne dépend pas du choix des coordonnées.

Pour $w \in \mathbb{P}\left(\mathbb{C}_{p}\right)$ on a

$$
\sum_{R(z)=w} \operatorname{deg}_{R}(z)=\operatorname{deg}(R)
$$

et pour $Q \in \mathbb{C}_{p}(z)$ on a $\operatorname{deg}_{Q \circ R}(w)=\operatorname{deg}_{Q}(R(w)) \cdot \operatorname{deg}_{R}(w)$.

Étant donnés $X, Y \subset \mathbb{P}\left(\mathbb{C}_{p}\right)$ tels que $R(X) \subset Y$, on dit que $R: X \rightarrow Y$ est $d e$ degré $d$, où $d \geq 1$, si pour tout $y \in Y$

$$
\sum_{x \in X, R(x)=y} \operatorname{deg}_{R}(x)=d
$$

De façon équivalente, tout point dans $Y$ a exactement $d$ antécédents dans $X$ comptés avec multiplicité. 
3.1. Action d'une fonction rationnelle sur les bouts. Fixons une fonction rationnelle $R \in \mathbb{C}_{p}(z)$ non constante.

Proposition 3.1. Soit $\mathcal{P}$ un bout rationnel (resp. irrationnel, singulier). Alors il existe un bout $\mathcal{P}^{\prime}$ de même nature et un entier $d \geq 1$ tel que pour toute chaîne évanescente $\left\{C_{i}\right\}_{i \geq 0}$ définissant $\mathcal{P}$, il existe $N \geq 1$ tel qu'on ait les propriétés suivantes.

1. $\left\{R\left(C_{i}\right)\right\}_{i \geq N}$ est une chaîne évanescente définissant $\mathcal{P}^{\prime}$.

2. Pour tout $i \geq N, R: C_{i} \rightarrow R\left(C_{i}\right)$ est de degréd.

Le bout $\mathcal{P}^{\prime}$ sera noté $R_{*}(\mathcal{P})$. De plus l'entier $d$ sera noté $\operatorname{deg}_{R}(\mathcal{P})$ et appelé degré local de $R$ en $\mathcal{P}$.

Lemme 3.2. Soit $\mathcal{P}$ un bout non singulier. Alors il existe un entier $N \geq 0$ tel qu'on ait les propriétés suivantes.

1. Chaque point $y \in B_{R_{*}(\mathcal{P})} a N+\operatorname{deg}_{R}(\mathcal{P})$ antécédents par $R$ dans $B_{\mathcal{P}}$.

2. Chaque point $y \notin B_{R_{*}(\mathcal{P})}$ a $N$ antécédents par $R$ dans $B_{\mathcal{P}}$.

En particulier, si $N=0$ alors $R\left(B_{\mathcal{P}}\right)=B_{R_{*}(\mathcal{P})}$ et $R: B_{\mathcal{P}} \rightarrow B_{R_{*}(\mathcal{P})}$ est de degré $\operatorname{deg}_{R}(\mathcal{P})$; si $N$ est strictement positif alors $R\left(B_{\mathcal{P}}\right)=\mathbb{P}\left(\mathbb{C}_{p}\right)$.

3.2. Action d'une fonction rationnelle sur $\mathbb{H}_{p}$. Pour chaque point $\& \in \mathbb{H}_{p}$ on définit un point $R_{*}(\delta) \in \mathbb{H}_{p}$ et un entier $\operatorname{deg}_{R}(\delta) \geq 1$ qu'on appelle degré local de $R$ en 8 .

Si $\delta=\{\mathcal{P}\} \in \mathbb{H}_{p}$ est un point singulier, on note $R_{*}(\delta)=\left\{R_{*}(\mathcal{P})\right\} \in \mathbb{H}_{p}$ et $\operatorname{deg}_{R}(\delta)=\operatorname{deg}_{R}(\mathcal{P}) \geq 1$. Si $\delta=\left\{\mathcal{P}, \mathcal{P}^{\prime}\right\} \in \mathbb{H}_{p}$ est un point irrationnel, alors $\operatorname{deg}_{R}(\mathcal{P})=\operatorname{deg}_{R}\left(\mathcal{P}^{\prime}\right) \geq 1$ et $\left\{R_{*}(\mathcal{P}), R_{*}\left(\mathcal{P}^{\prime}\right)\right\}$ est un point irrationnel de $\mathbb{H}_{p}$. On les note respectivement $\operatorname{deg}_{R}(\delta)$ et $R_{*}(\delta) \in \mathbb{H}_{p}$.

La proposition suivante décrit l'action d'une fonction rationnelle sur les points rationnels de $\mathbb{H}_{p}$; voir [R1], Proposition 2.4.

Proposition 3.3. Soit $R \in \mathbb{C}_{p}(z)$ une fonction rationnelle non constante et soit $s \in \mathbb{H}_{p}^{\mathbb{Q}}$ un point rationnel. Alors on a les propriétés suivantes.

1. Il existe un unique point rationnel $s^{\prime} \in \mathbb{H}_{p}^{\mathbb{Q}}$ tel que pour tout $\mathcal{P} \in s$ on a $R_{*}(\mathcal{P}) \in \mathcal{S}^{\prime}$.

2. Considérons des paramétrages

$$
\delta=\{\mathcal{P}(\xi)\}_{\xi \in \mathbb{P}\left(\overline{\mathbb{F}}_{p}\right)} \text { et } \mathcal{s}^{\prime}=\left\{\mathcal{P}^{\prime}(\xi)\right\}_{\xi \in \mathbb{P}\left(\overline{\mathbb{F}}_{p}\right)} .
$$

Alors il existe une fonction rationnelle $\widetilde{R} \in \overline{\mathbb{F}}_{p}(z)$ telle que pour tout $\xi \in \mathbb{P}\left(\overline{\mathbb{F}}_{p}\right)$ on ait

$$
R_{*}(\mathcal{P}(\xi))=\mathcal{P}^{\prime}(\widetilde{R}(\xi)) \text { et } \operatorname{deg}_{R}(\mathcal{P}(\xi))=\operatorname{deg}_{\widetilde{R}}(\xi) .
$$


Vol. 80 (2005)

Donc pour tout $\mathcal{P}^{\prime} \in \mathcal{S}^{\prime}$

$$
\sum_{\mathcal{P} \in S, R_{*}(\mathcal{P})=\mathcal{P}^{\prime}} \operatorname{deg}_{R}(\mathcal{P})=\operatorname{deg}(\widetilde{R}) .
$$

3. Il existe un sous-ensemble fini $\mathcal{T}$ de $\&$ tel que pour tout $\mathcal{P} \in \mathcal{T}$ on ait $R\left(B_{\mathcal{P}}\right)=$ $\mathbb{P}\left(\mathbb{C}_{p}\right)$ et tel que pour tout $\mathcal{P} \in S \backslash \mathcal{T}$ la image de $B_{\mathcal{P}}$ par $R$ soit $B_{R_{*}(\mathcal{P})}$ et l'application $R: B_{\mathcal{P}} \rightarrow B_{R_{*}(\mathcal{P})}$ soit de degré $\operatorname{deg}_{R}(\mathcal{P})$.

Le point $\S^{\prime}$ sera noté $R_{*}(\S)$. Le degré de $\widetilde{R}$ sera noté $\operatorname{deg}_{R}(\S)$ et ne dépend pas du choix des coordonnées.

\subsection{Action locale d'une fonction rationnelle}

Proposition 3.4. Soit $R \in \mathbb{C}_{p}(z)$ une fonction rationnelle non constante fixant $0 \in$ $\mathbb{P}\left(\mathbb{C}_{p}\right)$ et localement de la forme $R(z)=a_{d} z^{d}+a_{d+1} z^{d+1}+\cdots$, où $d=\operatorname{deg}_{R}(0)$. Alors pour $r>0$ petit on a

$$
R_{*}\left(\wp_{r}\right)=\wp_{\left|a_{d}\right| r^{d}} \quad \text { et } \quad \operatorname{deg}_{R}\left(\wp_{r}\right)=d=\operatorname{deg}_{R}(0),
$$

où $\diamond_{r} \in \mathbb{H}_{p}$ est le point associé à la boule $\{|z|<r\}$.

Proposition 3.5. Soit $R \in \mathbb{C}_{p}(z)$ une fonction rationnelle non constante. Soit $\mathcal{P}$ un bout et soit $s \in \mathbb{H}_{p}$ le point qui contient $\mathcal{P}$. Alors il existe un point $\bar{s} \in \mathbb{H}_{p}$ tel que $\bar{\delta} \prec B_{\mathcal{P}}$ et tel que l'on ait les propriétés suivantes.

1. $R_{*}((\delta, \bar{\delta}))=\left(R_{*}(\delta), R_{*}(\bar{\delta})\right)$ et $R_{*}$ est injective $\operatorname{sur}(\delta, \bar{\delta})$.

2. Pour tout $\delta^{\prime} \in(\delta, \bar{\gamma})$ on a $\operatorname{deg}_{R}\left(\mathcal{S}^{\prime}\right)=\operatorname{deg}_{R}(\mathcal{P})$.

3. $d\left(R_{*}(\S), R_{*}(\bar{\S})\right)=\operatorname{deg}_{R}(\mathcal{P}) \cdot d(\&, \bar{\S})$.

Corollaire 3.6. Soit $R \in \mathbb{C}_{p}(z)$ une fonction rationnelle non constante. Alors pour tout $\&$ et $\delta^{\prime} \in \mathbb{H}_{p}$ on a

$$
d\left(R_{*}(\S), R_{*}\left(\mathcal{\prime}^{\prime}\right)\right) \leq \operatorname{deg}(R) \cdot d\left(\&, \AA^{\prime}\right) .
$$

En particulier l'action sur $\mathbb{H}_{p}$ induite par une fonction rationnelle est continue.

Corollaire 3.7. Considérons un segment de $\mathbb{H}_{p}$ paramétré par $\left\{\delta_{t}\right\}_{0 \leq t \leq t^{\prime}}$, de telle façon que $d\left(\S_{t_{0}}, \wp_{t_{1}}\right)=\left|t_{0}-t_{1}\right|$ pour $0 \leq t_{0}<t_{1} \leq t^{\prime}$. Supposons que $R \in \mathbb{C}_{p}(z)$ soit une fonction rationnelle telle que $R_{*}$ est injective sur $\left[\delta_{0}, \delta_{t^{\prime}}\right]$. Alors

$$
d\left(R_{*}\left(\delta_{0}\right), R_{*}\left(\delta_{t^{\prime}}\right)\right)=\int_{0}^{t^{\prime}} \operatorname{deg}_{R}\left(\delta_{\tau}\right) d \tau .
$$

En particulier on a $d\left(R_{*}\left(\wp_{0}\right), R_{*}\left(\wp_{t^{\prime}}\right)\right) \geq d\left(\S_{0}, \S_{t^{\prime}}\right)$. 


\section{Fonctions rationnelles en caractéristique positive}

Dans ce paragraphe on considère des propriétés de fonctions rationnelles à coefficients dans $\overline{\mathbb{F}}_{p}$. Comme la caractéristique de $\overline{\mathbb{F}}_{p}$ est positive, parfois il y a des propriétés assez différentes du cas de caractéristique zéro ; par exemple le polynôme $z^{p} \in \overline{\mathbb{F}}_{p}[z]$ induit une bijection sur $\mathbb{P}\left(\overline{\mathbb{F}}_{p}\right)$.

Rappelons que pour toute puissance $q$ de $p$ on a $\overline{\mathbb{F}}_{p}=\bigcup_{n \geq 1} \mathbb{F}_{q^{n}}$.

Lemme 4.1. Soit $\widetilde{R} \in \overline{\mathbb{F}}_{p}(z)$ une fonction rationnelle.

1. Si $\operatorname{deg}(\widetilde{R})=1$ alors tout élément de $\mathbb{P}\left(\overline{\mathbb{F}}_{p}\right)$ est périodique par $\widetilde{R}$.

2. Si $\operatorname{deg}(\widetilde{R})>1$ alors tout élément de $\mathbb{P}(\overline{\mathbb{F}}$ p $)$ est prépériodique par $\widetilde{R}$ et $\widetilde{R}$ a une infinité de points périodiques.

Preuve. Soit $q \geq 1$ une puissance de $p$ telle que $\widetilde{R} \in \mathbb{F}_{q}(z)$. Alors pour chaque $n \geq 1$ l'ensemble $\mathbb{P}\left(\mathbb{F}_{q^{n}}\right)=\mathbb{F}_{q^{n}} \cup\{\infty\}$ est invariant par $\widetilde{R}$. Puisque $\mathbb{P}\left(\overline{\mathbb{F}}_{p}\right)=\bigcup_{n \geq 1} \mathbb{P}\left(\mathbb{F}_{q^{n}}\right)$, tout élément de $\mathbb{P}\left(\overline{\mathbb{F}}_{p}\right)$ est prépériodique par $\widetilde{R}$. Étant donné un entier $r \geq 0$ on désignera par $R^{r}$ la fonction rationnelle itérée $\underbrace{R \circ \cdots \circ R}_{r}$.

1. Si $\operatorname{deg}(\widetilde{R})=1$ alors $\widetilde{R}$ induit une bijection sur chaque $\mathbb{P}\left(\mathbb{F}_{q^{n}}\right)$ et par conséquent tout élément de $\mathbb{P}\left(\overline{\mathbb{F}}_{p}\right)$ est périodique par $\widetilde{R}$.

2. Supposons $\operatorname{deg}(\widetilde{R})>1$. Soient $\zeta_{1}, \ldots, \zeta_{k} \in \mathbb{P}\left(\overline{\mathbb{F}}_{p}\right)$ les points fixes de $\widetilde{R}$ et soient $\lambda_{1}, \ldots, \lambda_{k}$ leurs multiplicateurs. Pour chaque $1 \leq i \leq k$ tel que $\lambda_{i} \neq 0$ soit $n_{i} \geq 1$ le plus petit entier tel que $\lambda_{i}^{n_{i}}=1$.

Soit $r$ un nombre premier strictement supérieur à $p$ et aux $n_{i}$. Soit $\zeta$ une racine de $\widetilde{R}^{r}(z)-z$. Si $\zeta=\zeta_{i}$ et $\lambda_{i}=0$, alors $\zeta$ est une racine simple de $\widetilde{R}^{r}(z)-z$. Si $\zeta=\zeta_{i}, \lambda_{i} \neq 0$ et $n_{i}>1$, il en est de même (car $r$ n'est pas un multiple de $n_{i}$ ). Si $\zeta=\zeta_{i}, \lambda_{i} \neq 0$ et $n_{i}=1$, alors les multiplicités de $\zeta_{i}$ comme racine de $\widetilde{R}(z)-z$ et $\widetilde{R}^{r}(z)-z$ sont les mêmes. Il existe donc une racine $\zeta$ de $\widetilde{R}^{r}(z)-z$ distincte des $\zeta_{i}$ (car $\left.\operatorname{deg}\left(\widetilde{R}^{r}(z)-z\right)>\operatorname{deg}(\widetilde{R}(z)-z)\right)$. C'est un point périodique de période minimale $r$.

\subsection{Inséparabilité}

Définition 4.2. On dit qu'une fonction rationnelle $\widetilde{R} \in \overline{\mathbb{F}}_{p}(z)$ est inséparable s'il existe une fonction rationnelle $\widetilde{Q} \in \overline{\mathbb{F}}_{p}(z)$ telle que $\widetilde{R}(z)=\widetilde{Q}\left(z^{p}\right)$.

Notons que l'application $z \mapsto z^{p}$ est un automorphisme du corps $\overline{\mathbb{F}}_{p}$. Par conséquent pour toute fonction rationnelle $\widetilde{Q} \in \overline{\mathbb{F}}_{p}(z)$ il existe une fonction rationnelle $\widetilde{Q}_{1} \in \overline{\mathbb{F}}_{p}(z)$ telle qu'on ait $\widetilde{Q}\left(z^{p}\right)=\left(\widetilde{Q}_{1}(z)\right)^{p}$. On conclut alors que, si $\widetilde{R}$ appartient 
à $\overline{\mathbb{F}}_{p}(z)$ et $\widetilde{\varphi}, \widetilde{\psi}$ sont des transformations projectives de $\mathbb{P}\left(\overline{\mathbb{F}}_{p}\right)$, alors $\widetilde{R}$ est inséparable si et seulement si $\widetilde{\varphi} \circ \widetilde{R} \circ \widetilde{\psi}$ l'est.

Étant donnée une fonction rationnelle $\widetilde{R} \in \overline{\mathbb{F}}_{p}(z)$, on dit que $\zeta \in \mathbb{P}\left(\overline{\mathbb{F}}_{p}\right)$ est un point critique de $\widetilde{R} \operatorname{si} \operatorname{deg}_{\widetilde{R}}(\zeta)>1$. Dans ce cas on dit que $\operatorname{deg}_{\widetilde{R}}(\zeta)-1$ est la multiplicité de $\zeta$ comme point critique de $\widetilde{R}$.

La propriété suivante découle de la formule de Riemann-Hurwitz (voir par exemple [Ha], page 301, Collorary 2.4). Elle n'est pas difficile à montrer directement.

Proposition 4.3. Soit $\widetilde{R} \in \overline{\mathbb{F}}_{p}(z)$ une fonction rationnelle ayant plus de $2 \operatorname{deg}(\widetilde{R})-2$ points critiques, comptés avec multiplicité. Alors $\widetilde{R}$ est inséparable.

Le corollaire suivant est immédiat. En effet il découle du résultat plus faible que, si $\widetilde{R} \in \overline{\mathbb{F}}_{p}(z)$ est une fonction rationnelle qui n'est pas inséparable, alors l'ensemble de ces points critiques est fini.

Corollaire 4.4. Soit $\widetilde{R} \in \overline{\mathbb{F}}_{p}(z)$ une fonction rationnelle qui n'est pas inséparable. Alors il existe une infinité des points périodiques $\zeta \in \mathbb{P}\left(\overline{\mathbb{F}}_{p}\right)$ tels que $\operatorname{deg}_{\widetilde{R}^{n}}(\zeta)=1$, où $n \geq 1$ est la période de $\zeta$.

\section{Points périodiques dans $\mathbb{H}_{p}$}

Fixons une fonction rationnelle non constante $R \in \mathbb{C}_{p}(z)$ et considérons l'action $R_{*}$ sur $\mathbb{H}_{p}$ induite par $R$. On dit qu'un point $\delta \in \mathbb{H}_{p}$ est périodique par $R_{*}$ s'il existe $n \geq 1$ tel que $R_{*}^{n}(\S)=\S$. Dans ce cas on dit que $\delta$ est indifférent si $\operatorname{deg}_{R^{n}}(\S)=1$, et on dit que $\delta$ est répulsif si $\operatorname{deg}_{R^{n}}(\&)>1$.

Dans le paragraphe 5.2 on introduit les points périodiques inséparables, qui seront très importants dans ce qui suit.

5.1. Points périodiques indifférents. Pour une démonstration de la proposition suivante voir [R2], Proposition 5.8.

Proposition 5.1. Soit $R \in \mathbb{C}_{p}(z)$ une fonction rationnelle de degré au moins deux. Alors les propriétés suivantes sont équivalentes.

1. $R_{*}$ a un point périodique indifférent dans $\mathbb{H}_{p}$.

2. $R_{*}$ a une infinité de points périodiques indifférents dans $\mathbb{H}_{p}$.

3. $R$ a un point périodique indifférent dans $\mathbb{P}\left(\mathbb{C}_{p}\right)$.

4. $R$ a une infinité de points périodiques indifférents dans $\mathbb{P}\left(\mathbb{C}_{p}\right)$.

Dans ce cas $R_{*}$ possède aussi un point périodique répulsif dans $\mathbb{H}_{p}$.

On aura besoin de la proposition suivante dans le paragraphe 9.1. 
Proposition 5.2. Soit $R \in \mathbb{C}_{p}(z)$ une fonction rationnelle non constante et soit $\& \in \mathbb{H}_{p}$ un point irrationnel ou singulier fixé par $R_{*}$. Alors \& est indifférent et il est adhérent à l'ensemble des points fixes rationnels de $R_{*}$. En particulier tout point périodique répulsif de $R_{*}$ est rationnel.

Il est en fait vrai que l'ensemble des points périodiques indifférents dans $\mathbb{H}_{p}$ est ouvert, mais on n'aurait pas besoin de ce résultat plus fort. La démonstration de cette proposition s'appuie sur le lemme suivant.

Lemme 5.3. Soit $C$ une couronne et soit $R \in \mathbb{C}_{p}(z)$ une fonction rationnelle telle que $R(C)$ est aussi une couronne. Alors il existe un entier $d \geq 1$ tel que $R: C \rightarrow R(C)$ est de degré $d$ et on a,

$$
\bmod (R(C))=d \cdot \bmod (C)
$$

Preuve. Après changement de coordonnée on suppose que $C=\left\{r_{0}<|z|<r_{1}\right\}$ et que $R(C)=\left\{r_{0}^{\prime}<|z|<r_{1}^{\prime}\right\}$. En particulier $R$ n'a pas de zéro ni de pôle sur $C$. On pose $R=P / Q$, où

$$
\begin{aligned}
& P(z)=a_{0}+a_{1} z+\cdots+a_{n} z^{n} \in \mathbb{C}_{p}[z] \text { et } \\
& Q(z)=b_{0}+b_{1} z+\cdots+b_{n^{\prime}} z^{n^{\prime}} \in \mathbb{C}_{p}[z] .
\end{aligned}
$$

Alors il existe $0 \leq k \leq n$ (resp. $0 \leq k^{\prime} \leq n^{\prime}$ ) tel que $\left|a_{i}\right| r_{0}^{i} \leq\left|a_{k}\right| r_{0}^{k}$ (resp. $\left.\left|b_{j}\right| r_{0}^{j} \leq\left|b_{k^{\prime}}\right| r_{0}^{k^{\prime}}\right)$ pour $0 \leq i \leq k$ (resp. $0 \leq j \leq k^{\prime}$ ) et $\left|a_{i}\right| r_{1}^{i} \leq\left|a_{k}\right| r_{1}^{k}$ (resp. $\left.\left|b_{j}\right| r_{1}^{j} \leq\left|b_{k^{\prime}}\right| r_{1}^{k^{\prime}}\right)$ pour $k \leq i \leq n\left(\right.$ resp. $\left.k^{\prime} \leq j \leq n^{\prime}\right)$.

Comme $R(C)=\left\{r_{0}^{\prime}<|z|<r_{1}^{\prime}\right\}$ on a $k \neq k^{\prime}$, et quitte à changer $R$ par $\frac{1}{R}$, on suppose $k>k^{\prime}$. Alors $R: C \rightarrow R(C)$ est de degré $d=k-k^{\prime}$. Remarquons d'autre part que $r_{0}^{\prime}=\left(\left|a_{k}\right| /\left|b_{k^{\prime}}\right|\right) r_{0}^{d}$ et $r_{1}^{\prime}=\left(\left|a_{k}\right| /\left|b_{k^{\prime}}\right|\right) r_{1}^{d}$. Par conséquent

$$
\bmod (R(C))=\log _{p}\left(r_{1}^{\prime} / r_{0}^{\prime}\right)=\log _{p}\left(r_{1}^{d} / r_{0}^{d}\right)=d \cdot \bmod (C) .
$$

Preuve de la Proposition 5.2. Cas \& irrationnel. Après changement de coordonnée, on suppose que $\&$ est le point associé à $\{|z|<r\}$. Posons $R=P / Q$, avec

$$
\begin{aligned}
& P(z)=a_{0}+a_{1} z+\cdots+a_{d} z^{d} \in \mathbb{C}_{p}[z] \text { et } \\
& Q(z)=b_{0}+b_{1} z+\cdots+b_{d^{\prime}} z^{d^{\prime}} \in \mathbb{C}_{p}[z] .
\end{aligned}
$$

Soit $0 \leq n \leq d$ (resp. $0 \leq n^{\prime} \leq d^{\prime}$ ) le plus petit entier qui maximise $\left|a_{n}\right| r^{n}$ (resp. $\left.\left|b_{n^{\prime}}\right| r^{n^{\prime}}\right)$. Comme $r \notin\left|\mathbb{C}_{p}^{*}\right|$ on a $\left|a_{i}\right| r^{i}<\left|a_{n}\right| r^{n}$ (resp. $\left.\left|b_{j}\right| r^{j}<\left|b_{n^{\prime}}\right| r^{n^{\prime}}\right)$ pour $i \neq n$ (resp. $j \neq n^{\prime}$ ). Donc $|R(z)|=\left|a_{n} / b_{n^{\prime}}\right||z|^{n-n^{\prime}}$ pour tout $z$ tel que $|z|$ est assez proche de $r$. Par suite, $\left|a_{n} / b_{n^{\prime}}\right| r^{n-n^{\prime}}=r$, et comme $r \notin\left|\mathbb{C}_{p}^{*}\right|$, on a $\operatorname{deg}_{R}(\mathcal{P})=n-n^{\prime}=1$. 
Par conséquent $\&$ est un point fixe indifférent et $R_{*}$ fixe le point associé à $\left\{|z|<r^{\prime}\right\}$ pour tout $r^{\prime}$ proche de $r$.

Cas $\&$ singulier. Soit $\left\{D_{i}\right\}_{i \geq 0}$ une chaîne évanescente définissant $\&$ et soit $r_{0}=$ $\lim _{i \rightarrow \infty} \operatorname{diam}\left(D_{i}\right)>0$. Pour $r>0$ on désigne par $D_{r} \subset \mathbb{C}_{p}$ la boule ouverte ou irrationnelle de diamètre $r$ telle que $\delta \prec D_{r}$. Notons que pour $r<r^{\prime}$ on a $D_{r} \subset D_{r^{\prime}}$.

Fixons $r_{1}>r_{0}$ assez proche de $r_{0}$ tel que $D_{r_{1}}$ ne contient pas de point fixe de $R$, tel que $R\left(D_{r_{1}}\right) \subset \mathbb{C}_{p}$ et tel que $R: D_{r_{1}} \rightarrow R\left(D_{r_{1}}\right)$ soit de degré $d=\operatorname{deg}_{R}(\delta)$. Pour $r \in\left(r_{0}, r_{1}\right)$ on a $R\left(D_{r}\right) \subset R\left(D_{r_{1}}\right) \subset \mathbb{C}_{p}$ et lorsque $r^{\prime} \in\left(r, r_{1}\right)$ on a $R\left(D_{r}\right) \subset R\left(D_{r^{\prime}}\right)$ (Lemme 3.2). On désigne par $\rho(r)$ le diamètre de la boule $R\left(D_{r}\right)$; on a $R\left(D_{r}\right)=$ $D_{\rho(r)}$. Comme $R_{*}(\&)=\&$, on a $\rho(r) \rightarrow r_{0}$ lorsque $r \rightarrow r_{0}$.

Par le choix de $r_{1}$, pour tout $r \in\left(r_{0}, r_{1}\right)$ l'application $R: D_{r} \rightarrow R\left(D_{r}\right)$ est de degré $d$. Par conséquent, si $r \notin\left|\mathbb{C}_{p}^{*}\right|$, alors l'image de la couronne $D_{r_{1}} \backslash D_{r}$ est la couronne $R\left(D_{r_{1}}\right) \backslash R\left(D_{r}\right)$, et l'application $R: D_{r_{1}} \backslash D_{r} \rightarrow R\left(D_{r_{1}}\right) \backslash R\left(D_{r}\right)$ est de degré $d$. Par le lemme précédent on a $\left(\rho\left(r_{1}\right) / \rho(r)\right)^{d}=r_{1} / r$. En faisant tendre $r$ vers $r_{0}$ on obtient $\rho\left(r_{1}\right)^{d}=r_{0}^{d}\left(r_{1} / r_{0}\right)$. Par conséquent on a $\rho\left(r_{1}\right) \geq r_{1}$ avec égalité si et seulement si $d=1$.

Supposons par l'absurde que $d>1$. D'après ce qui précède on a $\rho\left(r_{1}\right)>r_{1}$ et alors le Lemme 1.1 implique que $(R-\mathrm{id})\left(D_{r_{1}}\right)=R\left(D_{r_{1}}\right)=\left\{|z|<\rho\left(r_{1}\right)\right\}$. En particulier $D_{r_{1}}$ contient un point fixe de $R$. On obtient donc une contradiction et on conclut que $\operatorname{deg}_{R}(\mathcal{P})=d=1$. Par conséquent, pour $r \in\left(r_{0}, r_{1}\right)$ on a $\rho(r)=r$ et alors le point de $\mathbb{H}_{p}$ associé à la boule $D_{r}$ est fixé par $R_{*}$.

5.2. Points périodiques inséparables. Étant donnés une fonction rationnelle $R \in$ $\mathbb{C}_{p}(z)$ et un point rationnel $\& \in \mathbb{H}_{p}^{\mathbb{Q}}$, on dit que $R_{*}$ est inséparable en $\&$ si l'action de $R_{*}$ en $\delta$ est inséparable, c'est-à-dire si $\operatorname{deg}_{R}(\mathcal{P})>1$ pour tout (une infinité de) $\mathcal{P} \in \mathcal{S}$; voir Proposition 4.3.

Définition 5.4. Soit $R \in \mathbb{C}_{p}(z)$ une fonction rationnelle de degré au moins deux. On dit qu'un point périodique $\delta \in \mathbb{H}_{p}$ de $R_{*}$ de période $n \geq 1$ est inséparable si $\delta$ est rationnel et si $\left(R_{*}\right)^{n}$ est inséparable en $\&$.

Remarquons que tout point périodique inséparable est répulsif.

Proposition 5.5. Soit $R \in \mathbb{C}_{p}(z)$ une fonction rationnelle telle que $R_{*} n$ 'a pas de points périodiques indifférents dans $\mathbb{H}_{p}$. Alors tous les points périodiques de $R_{*}$ dans $\mathbb{H}_{p}$ sont inséparables.

La démontration de cette proposition s'appuie sur le lemme suivant.

Lemme 5.6. Soit $R \in \mathbb{C}_{p}(z)$ une fonction rationnelle et soit $\mathcal{P}$ un bout non singulier tel que $R\left(B_{\mathcal{P}}\right)=B_{\mathcal{P}}$. Alors : 
1. Si $\operatorname{deg}_{R}(\mathcal{P})=1$, alors tout point $\&$ de $\mathbb{H}_{p}$ tel que $\& \prec B_{\mathcal{P}}$ est périodique indifférent.

2. Si $\operatorname{deg}_{R}(\mathcal{P})>1$, alors la boule $B_{\mathcal{P}}$ contient un seul point périodique de $R$ dans $B_{\mathcal{P}}$, qui est un point fixe attractif.

Preuve. On pose $d=\operatorname{deg}_{R}(\mathscr{P})$. Après changement de coordonnée on suppose $B_{\mathcal{P}}=$ $\{|z|<r\}$. La restriction de $R$ à $\{|z|<r\}$ est alors donnée par une série de la forme $a_{0}+a_{1} z+\cdots$, où $\left|a_{i}\right| r^{i}<r$ pour $0 \leq i<d,\left|a_{d}\right| r^{d}=r$ et $\left|a_{j}\right| r^{j} \leq r$ pour $j>d$.

1. Supposons $d=1$ et soit $k$ tel que $\left|a_{1}^{k}-1\right|<1$. Étant donné $r_{0} \in\left(\left|a_{0}\right|, r\right)$ on pose $B_{0}=\left\{|z| \leq r_{0}\right\}$. Il suffit de montrer que $R^{k p^{m}}$ converge uniformément vers l'identité sur $B_{0}$ lorsque $m \rightarrow \infty$. Notons qu'on a $\left\|R^{k}(z)-z\right\|_{r_{0}}<r_{0}$. Plus généralement, on montrera que pour toute série $f$ convergente sur la boule $B_{0}$ et telle que $\|f(z)-z\|_{r_{0}}<r_{0}$, la suite $\left\{f^{p^{m}}\right\}_{m \geq 0}$ converge uniformément vers l'identité sur $B_{0}$, lorsque $m \rightarrow \infty$.

Soit $\gamma=\|f(z)-z\|_{r_{0}} / r_{0}<1$ et pour chaque entier $n \geq 1$ posons $T_{n}(z)=$ $f^{n}(z)-z$. Par récurrence il suffit de montrer que pour tout entier $\ell \geq 1$ on a

$$
\left\|T_{p \cdot \ell}\right\|_{r_{0}} \leq \max \{|p|, \gamma\} \cdot\left\|T_{\ell}\right\|_{r_{0}} .
$$

Notons que pour tous $\ell, n \geq 1$ on a

$$
T_{n \ell}=T_{\ell}+T_{\ell} \circ f+\cdots+T_{\ell} \circ f^{n-1} .
$$

Lorsque $\ell=1$, l'inégalité ultramétrique implique $\left\|T_{n}\right\|_{r_{0}} \leq\left\|T_{1}\right\|_{r_{0}}=\gamma r_{0}$. Par conséquent, si l'on fixe $z_{0} \in B_{0}$ et on pose $D=\left\{\left|z-z_{0}\right| \leq \gamma r_{0}\right\}$, alors pour tout $n \geq 1$ on a $f^{n}\left(z_{0}\right) \in D$ et le Lemme 1.2 implique qu'on a

$$
\begin{aligned}
\left|T_{\ell} \circ f^{n}\left(z_{0}\right)-T_{\ell}\left(z_{0}\right)\right| & \leq \operatorname{diam}\left(T_{\ell}(D)\right) \\
& \leq\left(\operatorname{diam}(D) / r_{0}\right) \cdot\left\|T_{\ell}\right\|_{r_{0}}=\gamma \cdot\left\|T_{\ell}\right\|_{r_{0}},
\end{aligned}
$$

d'où on obtient $\left\|T_{\ell} \circ f^{n}-T_{\ell}\right\|_{r_{0}} \leq \gamma \cdot\left\|T_{\ell}\right\|_{r_{0}}$. On a donc

$$
\begin{aligned}
\left\|T_{p \ell}\right\|_{r_{0}} & \leq\left\|p T_{\ell}\right\|_{r_{0}}+\left\|\sum_{0 \leq n \leq p-1} T_{\ell} \circ f^{n}-T_{\ell}\right\|_{r_{0}} \\
& \leq \max \{|p|, \gamma\} \cdot\left\|T_{\ell}\right\|_{r_{0}} .
\end{aligned}
$$

2. Supposons $d>1$. Dans ce cas on a $R^{\prime}(z)<1$ sur $\{|z|<r\}$, et l'équation $R(z)-z=0$ a une solution $z_{0}$ sur $\{|z|<r\}$; elle est un point fixe attractif de $R$. De plus, il est facile de voir que pour $|z|<r$ la suite $R^{n}(z)$ converge vers $z_{0}$ lorsque $n \rightarrow \infty$. Donc, $z_{0}$ est le seul point périodique de $R$ dans $\{|z|<r\}$.

Preuve de la Proposition 5.5. Soit $\delta \in \mathbb{H}_{p}$ un point périodique de $R_{*}$. Par hypothèse $\delta$ est répulsif, et par conséquent il est rationnel. Quitte à changer $R$ par un itéré, on suppose que $\&$ est fixé par $R_{*}$. 
Soit $\mathcal{T}$ l'ensemble fini des bouts $\mathcal{P} \in \delta$ tel que $R\left(B_{\mathcal{P}}\right) \neq B_{R_{*}(\mathcal{P})}$. Comme $\&$ est répulsif, il existe une infinité de cycles de bouts dans $\&$ (Lemme 4.1). Par conséquent il existe une infinité de bouts $\mathcal{P}$ tels que $\left(R_{*}\right)^{n}(\mathcal{P})=\mathcal{P}$ pour un certain $n$ et tels que $\left(R_{*}\right)^{i}(\mathcal{P}) \in \mathcal{S} \backslash \mathcal{T}$ pour tout $i \geq 0$. Dans ce cas on a $R^{n}\left(B_{\mathcal{P}}\right)=B_{\mathcal{P}}$. Or, le lemme précédent implique que $\operatorname{deg}_{R}(\mathcal{P})>1$, car $R_{*} \mathrm{n}$ 'a aucun point périodique indifférent. Comme ceci est vrai pour une infinité de bouts $\mathcal{P} \in \mathcal{S}$, on conclut que l'action de $R_{*}$ est inséparable en 8 .

\section{Bonne réduction}

En coordonnées homogènes une fonction rationnelle s'écrit sous la forme $\left[P_{0}, P_{1}\right]$, où $P_{0}$ et $P_{1} \in \mathbb{C}_{p}\left[z_{0}, z_{1}\right]$ sont des polynômes homogènes de même degré, égal au degré de la fonction rationnelle. Pour $\lambda \in \mathbb{C}_{p}^{*},\left[\lambda P_{0}, \lambda P_{1}\right]$ représente la même fonction rationnelle.

Étant donné un polynôme $P \in \mathcal{O}_{p}\left[z_{0}, z_{1}\right]$ on désigne par $\widetilde{P}$ sa projection dans $\overline{\mathbb{F}}_{p}\left[z_{0}, z_{1}\right]$.

Définition 6.1. Considérons une fonction rationnelle $R \in \mathbb{C}_{p}(z)$ donnée en coordonnées homogènes par $\left[P_{0}, P_{1}\right]$. Quitte à remplacer $P_{0}$ et $P_{1}$ par $\lambda P_{0}$ et $\lambda P_{1}$ respectivement, on suppose $P_{0}$ et $P_{1}$ à coefficients entiers et tel qu'au moins l'un des coefficients de $P_{0}$ ou de $P_{1}$ soit de norme égale à 1 .

Si $\widetilde{P}_{0}$ et $\widetilde{P}_{1}$ n'ont pas de racine commune sur $\overline{\mathbb{F}}_{p} \times \overline{\mathbb{F}}_{p}$ autre que $(0,0)$, alors on dit que $R$ a bonne réduction. Dans ce cas la fonction rationnelle $\widetilde{R} \in \overline{\mathbb{F}}_{p}(z)$, donnée en coordonnées homogènes $\operatorname{par}\left[\widetilde{P}_{0}, \widetilde{P}_{1}\right]$, satisfait $\operatorname{deg}(\widetilde{R})=\operatorname{deg}(R)$, et on dit que $\widetilde{R}$ est la réduction de $R$.

De façon équivalente, une fonction rationnelle a bonne réduction si et seulement si elle s'étende en un endomorphisme du schéma $\mathbb{P}_{\mathcal{O}_{p}}^{1}$. La notion de bonne réduction à été introduite par Morton et Silverman dans [MS], voir aussi le paragraphe 7, [Ben2] et [R1].

Il est facile de voir qu'une fonction rationnelle $R \in \mathbb{C}_{p}(z)$ admet bonne réduction si et seulement si le point canonique $\S_{\text {can }} \in \mathbb{H}_{p}$ est fixé par $R_{*}$ et $\operatorname{deg}_{R}\left(\wp_{\text {can }}\right)=$ $\operatorname{deg}(R)$, cf. [R1]. Dans le cas où ceci a lieu, la fonction rationnelle $R \in \mathbb{F}_{p}(z)$ coïncide avec la fonction rationnelle donnée par la Proposition 3.3.

La proposition suivante est une conséquence immédiate du Lemme 6.3 ci-dessous.

Proposition 6.2. Soit $R \in \mathbb{C}_{p}(z)$ une fonction rationnelle ayant bonne réduction. Alors pour toute boule $B$ associée à $\wp_{\mathrm{can}}$, l'ensemble $R(B)$ est une boule associée $\grave{a} \&_{\text {can }}$. 
Lemme 6.3. Soit $R \in \mathbb{C}_{p}(z)$ une fonction rationnelle non constante. Si \& $\in \mathbb{H}_{p}$ est tel que $\operatorname{deg}_{R}(\delta)=\operatorname{deg}(R)$, alors pour toute boule $B$ associée à $\&$, l'ensemble $R(B)$ est une boule associée à $R_{*}(\delta)$.

Preuve. L'assertion est triviale si $\&$ est singulier. On suppose désormais $\&$ non singulier. Étant donné un bout $\mathcal{P} \in R_{*}(\delta)$, soient $\mathcal{P}_{1}, \ldots, \mathcal{P}_{k} \in \delta$ toutes les antécédents de $R_{*}(\mathcal{P})$ par $R_{*}$ dans $\&$. D'après la Proposition 3.3 on a

$$
\sum_{1 \leq i \leq k} \operatorname{deg}_{R}\left(\mathcal{P}_{i}\right)=\operatorname{deg}_{R}(\S)=\operatorname{deg}(R) .
$$

Fixons un point $z_{0} \in B_{\mathcal{P}}$. Par le Lemme 3.2 la boule $B_{\mathcal{P}_{i}}$ contient au moins $\operatorname{deg}_{R}\left(\mathcal{P}_{i}\right)$ antécédents par $R$ comptés avec multiplicité, et l'égalité a lieu si et seulement si $R\left(B_{\mathcal{P}_{i}}\right)=B_{R_{*}\left(\mathcal{P}_{i}\right)}$. Puisque le nombre d'antécédents de $z_{0}$ par $R$, comptés avec multiplicité, est égal à deg $(R)$, l'équation (4) implique qu'on a $R\left(B_{\mathcal{P}_{i}}\right)=B_{R_{*}}\left(\mathcal{P}_{i}\right)$ pour $1 \leq i \leq k$.

Le reste de ce paragraphe est consacré à démontrer la proposition suivante.

Proposition 6.4. Soit $R \in \mathbb{C}_{p}(z)$ une fonction rationnelle ayant bonne réduction. Alors on a les propriétés suivantes.

1. Pour tout point $s \in \mathbb{H}_{p}$ on a

$$
d\left(R_{*}(\S), \wp_{\text {can }}\right) \geq d\left(\S, \wp_{\text {can }}\right) .
$$

2. Si la réduction de $R$ est inséparable, alors pour tout point $s \in \mathbb{H}_{p}$ distinct de $\wp_{\text {can }}$ l'inégalité précédente est stricte. En particulier $\wp_{\text {can }}$ est le seul point périodique de $R_{*}$ dans $\mathbb{H}_{p}$.

3. Si la réduction de $R$ est inséparable, alors tous les points périodiques de $R$ dans $\mathbb{P}\left(\mathbb{C}_{p}\right)$ sont attractifs.

Lemme 6.5. Soit $R \in \mathbb{C}_{p}(z)$ une fonction rationnelle et soit $B$ une boule ouverte ou irrationnelle telle que $B^{\prime}=R(B)$ soit une boule; on désigne par $d \geq 1$ le degré de $R: B \rightarrow B^{\prime}$. Soit $s \in \mathbb{H}_{p}$ le point associé à $B$ et soit $\tilde{s} \in \mathbb{H}_{p}$ un point tel que $\widetilde{s} \prec B$. Alors on a

$$
d\left(R_{*}(\tilde{\S}), R_{*}(\S)\right) \geq d(\tilde{\S}, \S),
$$

avec égalité si et seulement si $d=1$.

Preuve. Après changement de coordonnée au départ et à l'arrivée on suppose $B=$ $\{|z|<r\}$ et $B^{\prime}=\left\{|z|<r^{\prime}\right\}$. Soit $D \subset B$ une boule différente de $B$. Par le Lemme 1.2 on a

$$
\frac{1}{r^{\prime}} \operatorname{diam}(R(D)) \leq \frac{1}{r} \operatorname{diam}(D),
$$


avec égalité si et seulement si $d=1$. Ceci montre l'inégalité désirée dans le cas où le point $\tilde{\delta}$ est non singulier. Si $d=1$ on a l'égalité dans (5), et donc on obtient l'égalité désirée dans le cas où le point $\widetilde{\S}$ est non singulier. L'égalité dans le cas où $\widetilde{\S}$ est singulier découle du cas précédent par continuité.

Considérons le cas où $d>1$ et $\widetilde{\S}$ est singulier. Soit $\left\{D_{i}\right\}_{i \geq 1}$ une chaîne évanescente répresentant $\widetilde{s}$ telle que $D_{0} \subset B$ et $D_{0} \neq B$. Le Lemme 1.2 implique

$$
\frac{1}{r^{\prime}} \operatorname{diam}\left(R\left(D_{0}\right)\right)<\frac{1}{r} \operatorname{diam}\left(D_{0}\right),
$$

et pour $i \geq 1$,

$$
\frac{\operatorname{diam}\left(R\left(D_{i}\right)\right)}{\operatorname{diam}\left(R\left(D_{0}\right)\right)} \leq \frac{\operatorname{diam}\left(D_{i}\right)}{\operatorname{diam}\left(D_{0}\right)}
$$

Comme

$$
\begin{aligned}
d\left(R_{*}(\tilde{\S}), R_{*}(\S)\right) & =\lim _{i \rightarrow \infty}-\log _{p}\left(\frac{1}{r^{\prime}} \operatorname{diam}\left(R\left(D_{i}\right)\right)\right) \text { et } \\
d(\tilde{\S}, \S) & =\lim _{i \rightarrow \infty}-\log _{p}\left(\frac{1}{r} \operatorname{diam}\left(D_{i}\right)\right),
\end{aligned}
$$

on conclut que $d\left(R_{*}(\tilde{\S}), R_{*}(\S)\right)>d(\tilde{8}, \S)$.

Preuve de la Proposition 6.4. 1. et 2. Soit $\delta \in \mathbb{H}_{p}$ distinct de $\delta_{\text {can }}$ et soit $B$ la boule associée à $\delta_{\text {can }}$ telle que $\delta \prec B$. Comme l'ensemble $R(B)$ est une boule (Proposition 6.2), le Lemme 6.5 implique que $d\left(R_{*}(\S), \S_{\text {can }}\right) \geq d\left(\S, \S_{\text {can }}\right)$.

Si la réduction de $R$ est inséparable, alors $R: B \rightarrow R(B)$ est de degré au moins $p>1$, et le Lemme 6.5 implique que l'inégalité précédente est stricte.

3. Si $z_{0} \in \mathbb{P}\left(\mathbb{C}_{p}\right)$ est un point périodique de $R$ de période $n \geq 1$, alors la boule $B$ associée à $\delta_{\text {can }}$ qui contient $z_{0}$ satisfait $R^{n}(B)=B$. Le Lemme 5.6 implique ainsi que $z_{0}$ est attractif.

\section{Bonne réduction et l'ensemble exceptionnel}

Dans ce paragraphe on considère l'ensemble exceptionnel de l'action sur $\mathbb{H}_{p}$ induite par une fonction rationnelle. Cet ensemble est défini de façon analogue au cas complexe, voir par exemple [Mi]. On peut consulter aussi [Hs] pour l'ensemble exceptionnel dans $\mathbb{P}\left(\mathbb{C}_{p}\right)$ d'une fonction rationnelle à coefficients dans $\mathbb{C}_{p}$.

Définition 7.1. On dit qu'un point $\delta \in \mathbb{H}_{p}$ est un point exceptionnel de $R$ si l'ensemble $\bigcup_{n \geq 0} R_{*}^{-n}(\delta)$ est fini.

Notons que tout antécédent d'un point exceptionnel est aussi un point exceptionnel. 
Le but de ce paragraphe est de montrer le théorème suivant, qui est un énoncé plus précis du Théorème 4 de l'introduction.

Théorème 5 (Ensemble Exceptionnel). Soit $R \in \mathbb{C}_{p}(z)$ une fonction rationnelle de degré au moins deux et soit $R_{*}$ l'action sur $\mathbb{H}_{p}$ induite par $R$.

L'ensemble exceptionnel de $R_{*}$ contient au plus 1 point. Il est non vide si et seulement si, après changement de coordonnée, $R$ a bonne réduction. Inversement, si $R$ a bonne réduction, alors le point $\delta_{\mathrm{can}}$ est le seul point exceptionnel de $R_{*}$.

Dans la démonstration de ce théorème on montre qu'un point $\delta \in \mathbb{H}_{p}$ est exceptionnel pour $R_{*}$ si et seulement si $R_{*}(\delta)=\delta$ et $\operatorname{deg}_{R}(\delta)=\operatorname{deg}(R)$; dans ce cas le point $\delta$ est rationnel et $R$ a bonne réduction dans une coordonnée $\varphi$ telle que $\varphi_{*}(\delta)=\delta_{\text {can }}$.

En suivant la terminologie introduite dans [R1], on dit qu' une fonction rationnelle $R$ est simple s'il existe une coordonnée dans laquelle $R$ a bonne réduction. On obtient ainsi le corollaire immédiat suivant du théorème précédent.

Corollaire 7.2. Soit $R \in \mathbb{C}_{p}(z)$ une fonction rationnelle de degré au moins deux. Si $R$ n'est pas simple, alors pour tout point \& de $\mathbb{H}_{p}$, l'ensemble $\bigcup_{n \geq 0} R_{*}^{-n}(\S)$ est infini.

La démonstration du Théorème 5 est une conséquence de la Proposition 7.3 cidessous. Une autre conséquence de cette proposition est une démonstration « conceptuelle » du théorème de Benedetto, énoncé dans l'introduction.

Proposition 7.3. Soit $R \in \mathbb{C}_{p}(z)$ une fonction rationnelle de degré au moins 2 . Supposons que $E \subset \mathbb{H}_{p}$ est un ensemble fini tel que $R_{*}(E) \subset E$ et tel que l'on ait $\operatorname{deg}_{R}(\delta)=\operatorname{deg}(R)$ pour tout $\delta \in E$. Alors $E$ contient au plus un point.

Cette proposition est une conséquence immédiate du lemme suivant.

Lemme 7.4. Soit $R \in \mathbb{C}_{p}(z)$ une fonction rationnelle non constante. Si $\varsigma_{0}, \S_{1} \in \mathbb{H}_{p}$ satisfont $\operatorname{deg}_{R}\left(\wp_{0}\right)=\operatorname{deg}_{R}\left(\wp_{1}\right)=\operatorname{deg}(R)$, alors

$$
d\left(R_{*}\left(\wp_{0}\right), R_{*}\left(\wp_{1}\right)\right)=\operatorname{deg}(R) \cdot d\left(\wp_{0}, \wp_{1}\right) .
$$

Preuve. Notons que la Proposition 3.5 implique qu'on a $\operatorname{deg}_{R}(\delta)=\operatorname{deg}(R)$ pour tout $\delta \in\left(\delta_{0}, \wp_{1}\right)$ proche de $\delta_{0}$ ou de $\varsigma_{1}$. Donc, on peut supposer $\wp_{0}$ et $\varsigma_{1}$ non singuliers.

Pour $i \in\{0,1\}$, soit $B_{i}$ la boule associée à $\delta_{i}$ telle que $\delta_{1-i} \prec B_{i}$. Alors $C=$ $B_{0} \cap B_{1}$ est une couronne (Lemme 2.6$)$ et $\bmod (C)=d\left(\delta_{0}, \delta_{1}\right)$ (voir paragraphe 2.5). Par le Lemme 6.3, l'ensemble $B_{i}^{\prime}=R\left(B_{i}\right)$ est une boule. Comme $B_{0} \cap B_{1} \neq \emptyset$ et $B_{0} \cup B_{1}=\mathbb{P}\left(\mathbb{C}_{p}\right)$, les boules $B_{0}^{\prime}$ et $B_{1}^{\prime}$ satisfont les mêmes propriétés. Par conséquent $C^{\prime}=B_{0}^{\prime} \cap B_{1}^{\prime}$ est une couronne, avec $\bmod \left(C^{\prime}\right)=d\left(R_{*}\left(\delta_{0}\right), R_{*}\left(\delta_{1}\right)\right)$. 
Pour $i \in\{0,1\}$ on pose $D_{i}=\mathbb{P}\left(\mathbb{C}_{p}\right) \backslash B_{i}$ et $D_{i}^{\prime}=\mathbb{P}\left(\mathbb{C}_{p}\right) \backslash B_{i}^{\prime}$. Notons qu'on a $D_{1-i} \subset B_{i}$ et

$$
R^{-1}\left(D_{1-i}^{\prime}\right)=\mathbb{P}\left(\mathbb{C}_{p}\right) \backslash R^{-1}\left(B_{1-i}^{\prime}\right) \subset D_{1-i} \subset B_{i} .
$$

Comme $B_{i}^{\prime}=R\left(B_{i}\right)$ le Lemme 3.2 implique que le degré local de $R$ au bout de $B_{i}$ est égal à deg $(R)$. On a donc $R^{-1}\left(B_{i}^{\prime}\right)=B_{i}$ et $R^{-1}\left(D_{i}^{\prime}\right)=D_{i}$. Puisque $\mathbb{P}\left(\mathbb{C}_{p}\right)=$ $D_{0} \sqcup C \sqcup D_{1}$ et $\mathbb{P}\left(\mathbb{C}_{p}\right)=D_{0}^{\prime} \sqcup C^{\prime} \sqcup D_{1}^{\prime}$, on conclut que $R^{-1}\left(C^{\prime}\right)=C$. Par conséquent $R: C \rightarrow C^{\prime}$ est de degré $\operatorname{deg}(R)$, et le Lemme 5.3 implique

$$
\bmod \left(C^{\prime}\right)=\operatorname{deg}(R) \cdot \bmod (C) .
$$

On aura besoin de la propriété générale suivante, laquelle est démontrée dans l'Appendice A.

Proposition. Soit $R \in \mathbb{C}_{p}(z)$ une fonction rationnelle non constante. Alors pour tout point $\& \in \mathbb{H}_{p}$ l'ensemble $R_{*}^{-1}(\&) \subset \mathbb{H}_{p}$ des antécédents de $\&$ par $R_{*}$ est fini, non vide et on a

$$
\sum_{R_{*}^{-1}(\delta)} \operatorname{deg}_{R}\left(f^{\prime}\right)=\operatorname{deg}(R) .
$$

Preuve du Théorème 5. Soit $\& \in \mathbb{H}_{p}$ un point exceptionnel de $R_{*}$ et considérons l'ensemble fini $E=\bigcup_{i \geq 0} R_{*}^{-n}(\S)$. Alors $R^{-1}(E) \subset E$, et par conséquent

$$
\sum_{E} \# R_{*}^{-1}\left(f^{\prime}\right)=\# R_{*}^{-1}(E) \leq \# E .
$$

Comme $R_{*}$ est surjective, pour tout $\boldsymbol{\zeta}^{\prime} \in E$ on a\# $R_{*}^{-1}\left(\delta^{\prime}\right)=1$ et $\operatorname{deg}_{R}\left(\delta^{\prime}\right)=\operatorname{deg}(R)$, cf. proposition précédente. Donc $R_{*}(E)=E$ et la Proposition 7.3 implique alors que $E=\{\S\}$. En particulier $R_{*}(\S)=\&$, et comme $\operatorname{deg}_{R}(\S)=\operatorname{deg}(R)$, on conclut que $\delta$ est rationnel et que $R$ a bonne réduction dans une coordonnée $\varphi$ telle que $\varphi_{*}(\S)=\varsigma_{\text {can }}$; voir paragraphe 6 .

D'autre part, supposons que $R$ a bonne réduction. Alors, d'après le paragraphe 6 , on a $R_{*}\left(\delta_{\text {can }}\right)=\wp_{\text {can }}$, et $\operatorname{deg}_{R}\left(\delta_{\text {can }}\right)=\operatorname{deg}(R)$. La proposition précédente implique que $R_{*}^{-1}\left(\delta_{\text {can }}\right)=\delta_{\text {can }}$ et donc $\delta_{\text {can }}$ est un point exceptionnel de $R$.

Il reste à montrer qu'il existe au plus 1 point exceptionnel; supposons que $\&$ et $\delta^{\prime}$ sont des points exceptionnels de $R_{*}$. D'après ce qui précéde on a $\operatorname{deg}_{R}(\delta)=$ $\operatorname{deg}_{R}\left(\delta^{\prime}\right)=\operatorname{deg}(R)$ et l'ensemble $E=\left\{\delta, \delta^{\prime}\right\}$ satisfait $R_{*}(E)=E$. Donc, la Proposition 7.3 implique que $\delta=\delta^{\prime}$.

Notons que d'après le paragraphe 6 , le théorème de Benedetto (énoncé dans l'introduction) peut être reformulé comme suit : pour un entier positif $n$ on a $R_{*}^{n}\left(\delta_{\text {can }}\right)=$ $\wp_{\text {can }}$ et $\operatorname{deg}_{R^{n}}\left(\wp_{\text {can }}\right)=\operatorname{deg}\left(R^{n}\right)$ si et seulement si $R_{*}\left(\wp_{\text {can }}\right)=\wp_{\text {can }}$ et $\operatorname{deg}_{R}\left(\wp_{\text {can }}\right)=$ $\operatorname{deg}(R)$. 
Preuve du Théorème de Benedetto. Clairement, si $R$ a bonne réduction, alors il est de même pour tous les itérés de $R$.

D'autre part, supposons que $R^{n}$ a bonne réduction et posons

$$
E=\left\{\wp_{\mathrm{can}}, R_{*}\left(\wp_{\mathrm{can}}\right), \ldots, R_{*}^{n-1}\left(\wp_{\mathrm{can}}\right)\right\} \subset \mathbb{H}_{p} .
$$

Comme $R_{*}^{n}\left(\wp_{\text {can }}\right)=\wp_{\text {can }}$ on a $R_{*}(E)=E$. D'autre part on a

$$
\Pi_{E} \operatorname{deg}(\wp)=\operatorname{deg}_{R^{n}}\left(\S_{\text {can }}\right)=\operatorname{deg}\left(R^{n}\right)=\operatorname{deg}(R)^{n},
$$

d'où $\operatorname{deg}_{R}(\S)=\operatorname{deg}(R)$ pour tout $\delta \in E$; en particulier $\operatorname{deg}_{R}\left(\wp_{\text {can }}\right)=\operatorname{deg}(R)$. La Proposition 7.3 implique alors que $E=\left\{\S_{\text {can }}\right\}$, d'où $R_{*}\left(\delta_{\text {can }}\right)=\wp_{\text {can }}$.

\section{Sur le nombre de points périodiques dans $\mathbb{H}_{p}$}

Dans ce paragraphe on déduit les théorèmes 1 et 2 à partir du Lemme Principal ci-dessous. Ce lemme est démontré dans le paragraphe 11. Le Théorème 3 est une conséquence simple de [R2], sa démonstration est ci-dessous. Finalement, nous illustrons ces résultats à l'aide de quelques exemples développés à la fin de ce paragraphe.

Lemme Principal. Soit $R \in \mathbb{C}_{p}(z)$ une fonction rationnelle telle que $R_{*}$ ait un point périodique inséparable dans $\mathbb{H}_{p}$ qui ne soit pas exceptionnel. Alors $R_{*}$ a une infinité de points périodiques inséparables.

Pour les démonstrations des théorèmes 1,2 et 3 , considérons une fonction rationnelle $R \in \mathbb{C}_{p}(z)$ de degré égal à 1 . Il y a deux cas.

1. $R$ est conjuguée à $z \mapsto \lambda z$, avec $|\lambda|<1$. Dans ce cas $R_{*}$ n'a pas de points périodiques dans $\mathbb{H}_{p}$. D'autre part, $R$ a exactement deux points périodiques dans $\mathbb{P}\left(\mathbb{C}_{p}\right)$ : un point fixe attractif et un point fixe répulsif.

2. $R$ est conjuguée à $z \mapsto z+1$ ou à $z \mapsto \lambda z$, avec $|\lambda|=1$. Dans ce cas tout point de $\mathbb{H}_{p}$ est périodique par $R_{*}$.

Les assertions des théorèmes 1, 2 et 3 sont faciles à vérifier dans ce cas. Ainsi on se ramène au cas des fonctions rationnelles de degré au moins égal à 2.

Preuve du Théorème 1. Soit $R \in \mathbb{C}_{p}(z)$ une fonction rationnelle de degré supérieure ou égal à 2 ayant au moins 2 points périodiques dans $\mathbb{H}_{p}$. Si $R_{*}$ a un point périodique indifférent, alors $R_{*}$ en a une infinité (Proposition 5.1). On suppose donc que $R_{*}$ n'a aucun point périodique indifférent. Alors tous les points périodiques de $R_{*}$ sont répulsifs et aussi inséparables, voir Proposition 5.5.

Soit $\&$ un point périodique de $R_{*}$. Si $\&$ est exceptionnel, le Théorème 5 implique que, après changement de coordonnée, $R$ a bonne réduction. Par ce qui précéde 
cette réduction est inséparable, et la Proposition 6.4 implique que $\delta$ est le seul point périodique de $R_{*}$. Mais par hypothèse $R_{*}$ a au moins deux points périodiques dans $\mathbb{H}_{p}$. Donc $\&$ n'est pas exceptionnel et le Lemme Principal implique que $R_{*}$ a une infinité de points périodiques dans $\mathbb{H}_{p}$.

Preuve $d u$ Théorème 2. Dans le cas où, après changement de coordonnée, $R$ a bonne réduction inséparable, les assertions du théorème ont été démontrées dans la Proposition 6.4 .

Supposons inversement que $R_{*}$ a exactement un point périodique dans $\mathbb{H}_{p}$. Dans ce cas $R_{*}$ n'a aucun point périodique indifférent dans $\mathbb{H}_{p}$ (Proposition 5.1) et par conséquent le (seul) point périodique de $R_{*}$ dans $\mathbb{H}_{p}$ est inséparable (Proposition 5.5). Le Lemme Principal implique que c'est un point exceptionnel, et par le Théorème 5, après changement de coordonnée, la fonction rationnelle $R$ a bonne réduction qui est inséparable.

Preuve du Théorème 3. D'après [Ben1], $R$ a au moins un point fixe non répulsif dans $\mathbb{P}\left(\mathbb{C}_{p}\right)$, voir aussi l'introduction de [R2]. L'implication $\mathrm{a} \Rightarrow \mathrm{b}$ est donc une conséquence du Théorème A' de [R2]. L'implication $b \Rightarrow c$ est triviale.

Montrons $\mathrm{c} \Rightarrow \mathrm{a}$. Supposons par l'absurde que $R_{*}$ a un point périodique dans $\mathbb{H}_{p}$. D'après la Proposition 5.1, $R_{*}$ a un point périodique répulsif dans $\mathbb{H}_{p}$ et le Corollaire 5.6 de [R2] fournit la conclusion désirée.

Dans le cas où les conditions équivalentes $\mathrm{a}, \mathrm{b}$ et $\mathrm{c}$ sont satisfaites, le seul point périodique non répulsif de $R$ est un point fixe. Il est forcément attractif, car l'existence d'un point périodique indifférent implique l'existence d'une infinité de points périodiques indifférents ; voir Corollaire 5.17 de [R1] pour une démonstration de ce fait.

Exemple 8.1. Considérons le polynôme $Q(z)=z^{p}+p z^{d} \in \mathbb{C}_{p}[z]$ avec $d>p$. Le point $\S_{\text {can }} \in \mathbb{H}_{p}$ est fixé par $Q_{*}$ et $Q_{*}$ est inséparable en $\delta_{\text {can. }}$. De plus $\operatorname{deg}_{Q}\left(\wp_{\text {can }}\right)=$ $p<\operatorname{deg}(Q)$, donc par le Théorème 5 le point $\delta_{\text {can }}$ n'est pas un point exceptionnel de $Q_{*}$. Le Lemme Principal implique alors que l'action $Q_{*}$ a une infinité de points périodiques (inséparables) dans $\mathbb{H}_{p}$.

\section{Applications propres et points fixes}

Le but de ce paragraphe est de montrer un critère pour l'existence de points fixes (Proposition 9.3), qu'on utilisera dans la démonstration du Lemme Principal pour «produire » des points périodiques.

Fixons une fonction rationnelle $R \in \mathbb{C}_{p}(z)$. Étant donné un ouvert $\widehat{\mathcal{V}} \subset \mathbb{H}_{p}$ on dit que l'application $R_{*}: \widehat{\mathcal{V}} \rightarrow R_{*}(\widehat{\mathcal{V}})$ est propre si $R_{*}(\partial \widehat{\mathcal{V}}) \cap R_{*}(\widehat{\mathcal{V}})=\emptyset$. 
Lemme 9.1. Soit $\widehat{\mathcal{V}} \subset \mathbb{H}_{p}$ un ouvert tel que l'application $R_{*}: \widehat{\mathcal{V}} \rightarrow R_{*}(\widehat{\mathcal{V}})$ soit propre. Soit $\widehat{W}^{\prime} \subset R_{*}(\widehat{\mathcal{V}})$ un ouvert connexe et soit $\widehat{\mathcal{W}} \subset \widehat{\mathcal{V}}$ une composante connexe de $R_{*}^{-1}\left(\widehat{\mathcal{W}}^{\prime}\right) \cap \widehat{\mathcal{V}}$. Alors $R_{*}(\widehat{\mathcal{W}})=\widehat{\mathcal{W}}^{\prime}$ et l'application $R_{*}: \widehat{\mathcal{W}} \rightarrow R_{*}(\widehat{\mathcal{W}})$ est propre.

Par exemple $R_{*}$, vue comme une application de $\mathbb{H}_{p}$ dans lui même, est propre. Comme conséquence du lemme, pour tout ouvert $\widehat{W} \subset \mathbb{H}_{p}$ et toute composante connexe $\widehat{W}_{0}$ de $R_{*}^{-1}(\widehat{W})$, on a $R_{*}\left(\widehat{W}_{0}\right)=\widehat{W}$ et l'application $R_{*}: \widehat{W}_{0} \rightarrow \widehat{W}$ est propre.

La démonstration du Lemme 9.1 dépend du lemme ci-dessous.

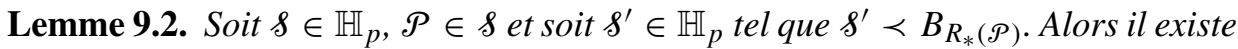
$\bar{s} \in R_{*}^{-1}\left(\delta^{\prime}\right)$ tel que $\bar{s} \prec B_{\mathcal{P}}$, tel que $R_{*}((\delta, \bar{\gamma}))=\left(R_{*}(\delta), \delta^{\prime}\right)$ et tel que $R_{*}$ soit injective sur $[\S, \bar{\delta}]$. En particulier

$$
d(\S, \bar{\S}) \leq d\left(R_{*}(\wp), \wp^{\prime}\right) .
$$

Preuve. La dernière assertion découle du Corollaire 3.7.

Posons $r_{0}=d\left(R_{*}(\delta), \delta^{\prime}\right)$ et pour $0 \leq r \leq r_{0}$ soit $\delta_{r}^{\prime} \in\left[R_{*}(\delta), \delta^{\prime}\right]$ le point tel que $d\left(R_{*}(\delta), \wp_{r}^{\prime}\right)=r$. En particulier $\delta_{0}^{\prime}=R_{*}(\delta)$ et $\delta_{r_{0}}^{\prime}=\delta^{\prime}$.

Posons $\wp_{0}=\S$. Il suffit de montrer que pour $0<r \leq r_{0}$ on peut définir $\wp_{r} \in \mathbb{H}_{p}$ tel qu'on ait $\wp_{r} \prec B_{\mathcal{P}}, R_{*}\left(\wp_{r}\right)=\wp_{r}^{\prime}$ et $\bigcup_{0 \leq r^{\prime} \leq r}\left\{\wp_{r^{\prime}}\right\}=\left[\wp_{0}, \wp_{r}\right]$. Ceci est une conséquence des considérations suivantes.

1. Supposons que $\wp_{r}$ est déjà défini pour $0 \leq r \leq r_{1}<r_{0}$. Soit $\mathcal{P}^{\prime} \in \wp_{r_{1}}^{\prime}=R_{*}\left(\wp_{r_{1}}\right)$ le bout tel que $\delta_{r_{0}}^{\prime} \prec B_{\mathcal{P}^{\prime}}$ et soit $\mathcal{P}_{1} \in \delta_{r_{1}}$ un bout tel que $R_{*}\left(\mathcal{P}_{1}\right)=\mathscr{P}^{\prime}$; si $r_{1}=0$ on choisit $\mathcal{P}_{1}=\mathcal{P}$. Par la Proposition 3.5 il existe $\varepsilon>0$ tel qu'on peut définir $\delta_{r} \in \widehat{B}_{\mathcal{P}_{1}}$ pour $r_{1} \leq r \leq r_{1}+\varepsilon$.

2. Supposons que $\S_{r}$ est déjà défini pour $0 \leq r<r_{1}$. Alors pour $0<r<r^{\prime}<r_{1}$ on a $d\left(\delta_{r}, \delta_{r^{\prime}}\right) \leq r^{\prime}-r$ (Proposition 3.5). Comme $\mathbb{H}_{p}$ est complet (paragraphe 2.5), il existe un point limite de $\left\{\ell_{r}\right\}_{0 \leq r<r_{1}}$, lorsque $r \rightarrow r_{1}$. On définit $\oiint_{r_{1}}$ comme ce point limite.

Preuve du Lemme 9.1. Fixons un point $s \in \widehat{W}$ et soit $\delta^{\prime} \in \widehat{W}^{\prime} \backslash\left\{R_{*}(\delta)\right\}$. Par le Lemme 9.2 il existe $\bar{s} \in R_{*}^{-1}\left(\delta^{\prime}\right)$ tel que $R_{*}((\&, \bar{s}))=\left(R_{*}(\S), \varsigma^{\prime}\right) \subset \widehat{W}^{\prime} \subset R_{*}(\widehat{\mathcal{V}})$. Comme l'application $R_{*}: \widehat{\mathcal{V}} \rightarrow R_{*}(\widehat{\mathcal{V}})$ est propre on a $[\delta, \bar{\delta}] \subset \widehat{\mathcal{V}}$. Donc $\bar{\delta} \in \widehat{\mathcal{W}}$ et par conséquent $R_{*}(\widehat{\mathcal{W}})=\widehat{W}^{\prime}$.

D'autre part soit $\bar{\delta} \in \partial \widehat{\mathcal{W}}$. Si de plus $\bar{\delta} \in \partial \widehat{\mathcal{V}}$ alors $R_{*}(\bar{\delta}) \notin \widehat{\mathcal{W}}^{\prime} \subset R_{*}(\widehat{\mathcal{V}})$, car $R_{*}: \widehat{\mathcal{V}} \rightarrow R_{*}(\widehat{\mathcal{V}})$ est propre. Si $\bar{\delta} \in \widehat{\mathcal{V}}$ alors $R_{*}(\bar{\delta}) \notin \widehat{W}^{\prime}$, par définition de $\widehat{\mathcal{W}}$. Donc $R_{*}: \widehat{W} \rightarrow \widehat{W}^{\prime}$ est propre. 


\subsection{Un critère pour l'existence de point fixe}

Proposition 9.3. Soit $R \in \mathbb{C}_{p}(z)$ une fonction rationnelle et soit $\widehat{\mathcal{V}} \subset \mathbb{H}_{p}$ un ouvert connexe tel que $R_{*}: \widehat{\mathcal{V}} \rightarrow R_{*}(\widehat{\mathcal{V}})$ soit propre et tel que $R_{*}(\widehat{\mathcal{V}})$ contient la fermeture topologique $\overline{\mathcal{V}}$ de $\widehat{\mathcal{V}}$. Alors, soit $\widehat{\mathcal{V}}$ contient un point fixe rationnel de $R_{*}$, soit il existe un point fixe répulsif $z_{0} \in \mathbb{P}\left(\mathbb{C}_{p}\right)$ de $R$ tel que $\widehat{\mathcal{V}}$ contient une demi-géodésique issue de $z_{0}$.

Le corollaire suivant est une conséquence immédiate de cette proposition.

Corollaire 9.4. Soit $\widehat{W} \subset \mathbb{H}_{p}$ un ouvert connexe borné et soit $\widehat{\mathcal{V}}$ une composante connexe de $R_{*}^{-1}(\widehat{W})$. Si $\widehat{W}$ contient la fermeture de $\widehat{\mathcal{V}}$, alors $\widehat{\mathcal{V}}$ contient un point fixe rationnel de $R_{*}$.

Preuve de la Proposition 9.3. Supposons que $R_{*}$ n'ait pas de point fixe rationnel dans $\widehat{\mathcal{V}}$. Alors $R_{*}$ n'a pas de point fixe dans $\widehat{\mathcal{V}}$, voir Proposition 5.2.

1 . Fixons $\varsigma_{0} \in \widehat{\mathcal{V}}$. Pour chaque $t \geq 0$ on définira $\varsigma_{t} \in \widehat{\mathcal{V}}$ à distance $t$ de $\delta_{0}$ tel que $R_{*}$ soit injective sur $\left[\wp_{0}, \wp_{t}\right]$, tel que pour tout $\delta \in\left[\wp_{0}, \wp_{t}\right]$ on ait $R_{*}(\delta) \in\left[R_{*}\left(\wp_{0}\right), \delta\right)$ et tel que

$$
\left[\S_{0}, \wp_{t}\right]=\bigcup_{0 \leq t^{\prime} \leq t}\left\{\oiint_{t^{\prime}}\right\} \quad \text { et } \quad\left[R_{*}\left(\wp_{0}\right), R_{*}\left(\wp_{t}\right)\right]=\bigcup_{0 \leq t^{\prime} \leq t}\left\{R_{*}\left(\wp_{t^{\prime}}\right)\right\} \text {. }
$$

1.1. Soit $t_{0} \geq 0$ tel que $\delta_{t}$ est déjà défini pour $0 \leq t \leq t_{0}$. On définira $\delta_{t}$ pour $t>t_{0}$ proche de $t_{0}$.

Notons que par hypothèse $R_{*}\left(\S_{t_{0}}\right) \in\left[R_{*}\left(\S_{0}\right), \S_{t_{0}}\right)$, et donc

$$
\left[R_{*}\left(\delta_{0}\right), \delta_{t_{0}}\right)=\left[R_{*}\left(s_{0}\right), R_{*}\left(s_{t_{0}}\right)\right] \sqcup\left(R_{*}\left(\wp_{t_{0}}\right), \S_{t_{0}}\right) .
$$

Soit $\bar{\delta} \in R_{*}^{-1}\left(\delta_{t_{0}}\right)$ donné par le Lemme 9.2, de telle façon que $R_{*}$ soit injective $\operatorname{sur}\left[\delta_{t_{0}}, \bar{\delta}\right]$ et $R_{*}\left(\left(\wp_{t_{0}}, \bar{\delta}\right)\right)=\left(R_{*}\left(\wp_{t_{0}}\right), \wp_{t_{0}}\right)$. Comme $R_{*}$ est injective sur $\left[\wp_{0}, \wp_{t_{0}}\right]$ et $R_{*}\left(\left[\delta_{0}, \delta_{t_{0}}\right]\right)=\left[R_{*}\left(\wp_{0}\right), R_{*}\left(\wp_{t_{0}}\right)\right]$, on a $\delta_{t_{0}} \in\left(\delta_{0}, \bar{\delta}\right)$ et $R_{*}$ est injective $\operatorname{sur}\left(\wp_{0}, \bar{\gamma}\right)$.

Soit $t_{1}>t_{0}$ assez proche de $t_{0}$ tel que $\left[\delta_{t_{0}}, \delta_{t_{1}}\right] \subset \widehat{\mathcal{V}}$ et tel que

$$
d\left(\delta_{t_{0}}, \wp_{t_{1}}\right)+d\left(R_{*}\left(\delta_{t_{0}}\right), R_{*}\left(\delta_{t_{1}}\right)\right)<d\left(\S_{t_{0}}, R_{*}\left(\delta_{t_{0}}\right)\right),
$$

où $\delta_{t_{1}} \in\left(\S_{0}, \bar{\delta}\right)$ est le point à distance $t_{1}$ de $\delta_{0}$, voir figure 1 . Pour $t_{0}<t<t_{1}$ soit $\wp_{t}$ le point dans $\left[\wp_{t_{0}}, \wp_{t_{1}}\right]$ à distance $t$ de $\wp_{0}$.

Il reste à montrer que pour tout $t_{0}<t \leq t_{1}$ on a $R_{*}\left(\delta_{t}\right) \in\left[R_{*}\left(\delta_{0}\right), \wp_{t}\right)$. Comme $R_{*}\left(\delta_{t}\right) \in\left(R_{*}\left(\delta_{t_{0}}\right), \delta_{t_{0}}\right)$, les bouts $\mathcal{P}, \mathcal{P}^{\prime} \in R_{*}\left(\delta_{t}\right)$ tels que $s_{t_{0}} \prec B_{\mathcal{P}}$ et $R_{*}\left(\delta_{t_{0}}\right) \prec$ $B_{\mathcal{P}^{\prime}}$ respectivement, sont distincts. Alors $\S_{t} \prec B_{\mathcal{P}}$, car sinon $R_{*}\left(\delta_{t}\right) \in\left(\S_{t_{0}}, \wp_{t}\right]$ et on aurait

$$
\begin{aligned}
& d\left(\wp_{t_{0}}, \wp_{t}\right) \geq d\left(\wp_{t_{0}}, R_{*}\left(\wp_{t}\right)\right) \geq d\left(\S_{t_{0}}, R_{*}\left(\wp_{t_{1}}\right)\right) \\
& =d\left(\delta_{t_{0}}, R_{*}\left(\delta_{t_{0}}\right)\right)-d\left(R_{*}\left(\delta_{t_{0}}\right), R_{*}\left(\delta_{t_{1}}\right)\right)>d\left(\delta_{t_{0}}, \wp_{t_{1}}\right) \text {, }
\end{aligned}
$$


où la dernière inégalité découle de (6). Donc $\S_{t} \prec B_{\mathcal{P}}$ et comme $R_{*}\left(\S_{0}\right) \prec B_{\mathcal{P}^{\prime}}$ on a $R_{*}\left(\wp_{t}\right) \in\left(R_{*}\left(\delta_{0}\right), \S_{t}\right)$.

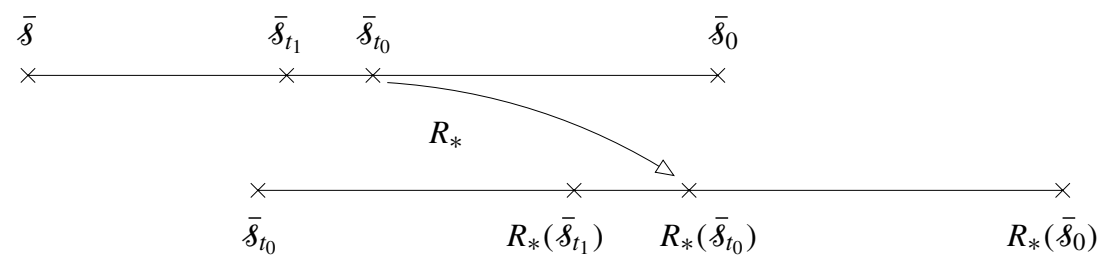

Figure 1

1.2. Soit $t_{0}>0$ et supposons que $\oiint_{t}$ est déjà défini pour $0 \leq t<t_{0}$. On va définir $S_{t_{0}}$.

Comme $\mathbb{H}_{p}$ est complet (paragraphe 2.5), il existe un point $\delta_{t_{0}} \in \overline{\mathcal{V}}$ tel que $\left[\delta_{0}, \delta_{t_{0}}\right)=\bigcup_{0 \leq t<t_{0}}\left\{\wp_{t}\right\}$. En particulier, $\delta_{t_{0}}$ est à distance $t_{0}$ de $\delta_{0}, R_{*}$ est injective sur $\left[\delta_{0}, \wp_{t_{0}}\right]$ et par continuité on a $R_{*}\left(\delta_{t_{0}}\right) \in\left[R_{*}\left(\wp_{0}\right), \wp_{t_{0}}\right]$. Il reste à montrer que $R_{*}\left(\delta_{t_{0}}\right) \in\left[R_{*}\left(\delta_{0}\right), \delta_{t_{0}}\right)$.

Supposons par l'absurde que $R_{*}\left(\delta_{t_{0}}\right)=\wp_{t_{0}}$. On a donc $R_{*}\left(s_{t_{0}}\right)=\delta_{t_{0}} \in \overline{\mathcal{V}} \subset$ $R_{*}(\widehat{\mathcal{V}})$. Comme $\left[s_{0}, s_{t_{0}}\right) \subset \widehat{\mathcal{V}}$ et comme l'application $R_{*}: \widehat{\mathcal{V}} \rightarrow R_{*}(\widehat{\mathcal{V}})$ est propre, on a $R_{*}(\partial \widehat{\mathcal{V}}) \cap R_{*}(\widehat{\mathcal{V}})=\emptyset$, d'où on conclut que $s_{t_{0}} \in \widehat{\mathcal{V}}$. On obtient une contradiction, car par hypothèse $R_{*}$ n'a aucun point fixe dans $\widehat{\mathcal{V}}$.

2. Comme conséquence de 1.1 et 1.2 , on peut définir $s_{t} \in \widehat{\mathcal{V}}$, pour $t \geq 0$, satisfaisant les propriétés dans 1 . Pour $t \geq 0$ soit $\mathcal{P}_{t} \in \vartheta_{t}$ le bout tel que $\delta_{t^{\prime}} \in \widehat{B}_{\mathcal{P}_{t}}$ pour $t^{\prime}>t$.

Alors pour $t<t^{\prime}$ la boule $B_{\mathcal{P}_{t}}$ contient $B_{\mathcal{P}_{t^{\prime}}}$ strictement. Comme $\delta_{t}$ est à distance $t$ de $s_{0}$, le diamètre de $B_{\mathcal{P}_{t}}$ tend vers zéro lorsque $t \rightarrow \infty$. Comme $\mathbb{P}\left(\mathbb{C}_{p}\right)$ est complet, il existe $z_{0} \in \mathbb{P}\left(\mathbb{C}_{p}\right)$ tel que $\cap_{t \geq 0} B_{\mathcal{P}_{t}}=\left\{z_{0}\right\}$. La propriété $R_{*}\left(\wp_{t}\right) \in\left[R_{*}\left(\wp_{0}\right), \S_{t}\right)$ implique que $z_{0}$ est un point fixe de $R$. La propriété $R_{*}\left(\wp_{t}\right) \in\left[R_{*}\left(\wp_{0}\right), \delta_{t}\right)$ implique aussi que $z_{0}$ est répulsif (cf. Proposition 3.4).

\section{Lemme d'Approximation et inséparabilité}

Dans ce paragraphe on s'occupe des propriétés générales qui sont indépendantes de la dynamique. Notamment, on montre que la propriété locale d'inséparabilité se traduit géométriquement en une expansion locale (Lemme 10.3).

10.1. Lemme d'Approximation. Soient $R$ et $Q \in \mathbb{C}_{p}(z)$ deux fonctions rationnelles ayant la même réduction non triviale, c'est-à-dire que

$$
R_{*}\left(\wp_{\text {can }}\right)=Q_{*}\left(\wp_{\text {can }}\right)=\wp_{\text {can }}
$$


et $R_{*}$ et $Q_{*}$ coüncident sur $\S_{\mathrm{can}}$. Alors $R_{*}$ et $Q_{*}$ coüncident sur un voisinage de $\S_{\mathrm{can}}$ dans $\mathbb{H}_{p}$.

La démonstration de ce lemme s'appui sur le lemme suivant.

Lemme 10.1. Soit $R \in \mathbb{C}_{p}(z)$ une fonction rationnelle telle que $R_{*}\left(s_{\text {can }}\right)=s_{\text {can }}$ et soit $X$ un affinoüde ouvert tel que $s_{\text {can }} \prec X$ et $R(X) \subset \mathbb{C}_{p}$. Alors pour toute fonction rationnelle $\varepsilon \in \mathbb{C}_{p}(z)$ telle qu'il existe $r_{0}<1$ satisfaisant $\varepsilon(X) \subset\left\{|z|<r_{0}\right\}$, les actions $R_{*}$ et $(R+\varepsilon)_{*}$ coüncident sur un voisinage de $\S_{\text {can }}$ dans $\mathbb{H}_{p}$.

Preuve. Considérons un point rationnel $s \in \mathbb{H}_{p}^{\mathbb{Q}}$ tel que $\delta \prec X$. Alors il existe une boule $B$ associée à $\&$ contenue dans $X$. Comme $R(B) \subset R(X) \subset \mathbb{C}_{p}$, l'ensemble $R(B)$ est une boule (Lemme 3.2).

Si $\&$ satisfait $d\left(R_{*}(\&), \wp_{\text {can }}\right)<\log _{p} \frac{1}{r_{0}}$, alors la boule $R(B)$ a un diamètre plus grand que $r_{0}$, et comme $\varepsilon(B) \subset \varepsilon(X) \subset\left\{|z|<r_{0}\right\}$, on conclut que $(R+\varepsilon)(B)=$ $R(B)$ (Lemme 1.1). Par conséquent $(R+\varepsilon)_{*}(\delta)=R_{*}(\delta)$.

Comme $R_{*}$ et $(R+\varepsilon)_{*}$ sont continues (Corollaire 3.6), on conclut qu'elles coïncident sur l'ensemble

$$
\left\{s \in \mathbb{H}_{p} \mid \& \prec X, d\left(R_{*}(\delta), \S_{\mathrm{can}}\right)<\log _{p} \frac{1}{r_{0}}\right\},
$$

lequel est un voisinage de $\wp_{\text {can }}$ dans $\mathbb{H}_{p}$.

Preuve du Lemme d'Approximation. Posons $\varepsilon=Q-R \in \mathbb{C}_{p}(z)$. Soit $\mathcal{T} \subset s_{\text {can }}$ un ensemble fini tel que pour $\mathcal{P} \in s_{\text {can }} \backslash \mathcal{T}$ on ait $R\left(B_{\mathcal{P}}\right) \subset \mathbb{C}_{p}$ et $Q\left(B_{\mathcal{P}}\right) \subset \mathbb{C}_{p}$. Dans ce cas, $R\left(B_{\mathcal{P}}\right)=B_{R_{*}(\mathcal{P})}$ et $Q\left(B_{\mathcal{P}}\right)=B_{Q_{*}(\mathcal{P})}\left(\right.$ Lemme 3.2) et donc $R\left(B_{\mathcal{P}}\right)=$ $Q\left(B_{\mathcal{P}}\right) \subset\{|z| \leq 1\}$. Par conséquent $\varepsilon\left(B_{\mathcal{P}}\right)=(R-Q)\left(B_{\mathcal{P}}\right) \subset\{|z|<1\}$.

On suppose d'abord que la fonction rationnelle $\varepsilon$ n'est pas constante. Comme l'ensemble $\mathcal{T} \subset \S_{\text {can }}$ est fini, on peut trouver $\mathcal{P}_{0}, \mathcal{P}_{1} \in \wp_{\text {can }} \backslash \mathcal{T}$ tels que $\varepsilon_{*}\left(\mathcal{P}_{0}\right)$ et $\mathcal{E}_{*}\left(\mathcal{P}_{1}\right)$ soient distincts. Donc, $\varepsilon_{*}\left(\delta_{\text {can }}\right) \prec\{|z|<1\}$ et par conséquent il existe $r_{0} \in$ $(0,1)$ tel que $\varepsilon_{*}\left(\delta_{\text {can }}\right) \prec\left\{|z|<r_{0}\right\}$; pour tout $\mathcal{P} \in S_{\text {can }} \backslash \mathcal{T}$ on a $\varepsilon\left(B_{\mathcal{P}}\right) \subset\left\{|z|<r_{0}\right\}$. Pour chaque $\mathcal{P} \in \mathcal{T}$ soit $C_{\mathcal{P}} \subset B_{\mathcal{P}}$ une couronne de la forme $C_{\mathcal{P}}=B_{\mathcal{P}} \backslash B_{\mathcal{P}}^{\prime}$, où $B_{\mathcal{P}}^{\prime} \subset B_{\mathcal{P}}$ est une boule fermée, de telle façon que

$$
R\left(C_{\mathcal{P}}\right) \subset \mathbb{C}_{p} \text { et } \varepsilon\left(C_{\mathcal{P}}\right) \subset\left\{|z|<r_{0}\right\}
$$

Si la fonction rationnelle $\varepsilon$ est constante, égale à $c$, alors d'après ce qui précède on a $|c|<1$. On choisit alors $r_{0} \in(|c|, 1)$ et pour $\mathcal{P} \in \mathcal{T}$ on définit les couronnes $C_{\mathcal{P}}$ comme ci-dessus.

Notons que par construction l'affinoïde ouvert $X=\mathbb{P}\left(\mathbb{C}_{p}\right) \backslash \sqcup_{\mathcal{T}} B_{\mathcal{P}}^{\prime}$ satisfait $\varsigma_{\text {can }} \prec X, R(X) \subset \mathbb{C}_{p}$ et $\varepsilon(X) \subset\left\{|z|<r_{0}\right\}$. Le lemme découle alors du lemme précédent. 


\subsection{Expansion locale}

Proposition 10.2. Soit $R \in \mathbb{C}_{p}(z)$ une fonction rationnelle non constante. Alors, pour un point rationnel $s \in \mathbb{H}_{p}^{\mathbb{Q}}$, on a les propriétés suivantes.

1. $R_{*}$ est inséparable en $\&$ si et seulement s'il existe un voisinage $\widehat{\mathcal{V}}$ de $\&$ dans $\mathbb{H}_{p}$ tel que $\operatorname{deg}_{R}\left(\delta^{\prime}\right) \geq$ p pour tout $\delta^{\prime} \in \widehat{\mathcal{V}}$. Dans ce cas on conclut que $R_{*}$ est inséparable en tout point rationnel contenu dans $\widehat{\mathcal{V}}$.

2. Il existe un voisinage $\widehat{\mathcal{V}}$ de $\&$ dans $\mathbb{H}_{p}$ tel que pour tout point $\bar{\delta} \in \widehat{\mathcal{V}}$ on a $d\left(R_{*}(\bar{\S}), R_{*}(\S)\right) \geq d(\bar{\delta}, \S)$. Si $R_{*}$ est inséparable en $\delta$, alors on peut choisir $\widehat{\mathcal{V}}$ tel que pour tout point $\bar{\S}$ dans $\widehat{\mathcal{V}}$ on ait $d\left(R_{*}(\bar{\delta}), R_{*}(\S)\right) \geq p \cdot d(\bar{\delta}, \S)$.

Pour montrer cette proposition, notons qu'après changement de coordonnée au départ et à l'arrivée, on peut supposer $R_{*}(\delta)=\delta=\delta_{\text {can }}$. Alors la proposition est une conséquence immédiate du lemme suivant, voir aussi la Proposition 3.5.

Lemme 10.3. Soit $R \in \mathbb{C}_{p}(z)$ une fonction rationnelle ayant une réduction non triviale $\widetilde{R} \in \overline{\mathbb{F}}_{p}(z)$. Étant donné un entier $n \geq 0$, les propriétés suivantes sont équivalentes.

1. On a $\operatorname{deg}_{R}(\delta) \geq p^{n}$ pour tout point $\& \in \mathbb{H}_{p}$ dans un voisinage de $\S_{\text {can }}$.

2. On a $\operatorname{deg}_{R}(\mathcal{P}) \geq p^{n}$ pour tout (resp. pour une infinité de) $\mathcal{P} \in \S_{\text {can. }}$.

3. Il existe une fonction rationnelle $\widetilde{Q} \in \overline{\mathbb{F}}_{p}(z)$ telle que $\widetilde{R}(z)=\widetilde{Q}\left(z^{p^{n}}\right)$.

Dans ce cas, pour tout point $s \in \mathbb{H}_{p}$ dans un voisinage de $\varsigma_{\text {can }}$ on a

$$
d\left(R_{*}(\S), \S_{\text {can }}\right) \geq p^{n} \cdot d\left(\S, \wp_{\text {can }}\right) .
$$

Preuve. L'implication $3 \Rightarrow 2$ est triviale et l'implication $2 \Rightarrow 3$ découle de la Proposition 4.3, par récurrence sur $n \geq 1$. L'implication $1 \Rightarrow 2$ découle de la Proposition 3.5. Supposons que la propriété 3 soit vraie et montrons que la propriété 1 est satisfaite.

Soit $Q \in \mathbb{C}_{p}(z)$ une fonction rationnelle ayant $\widetilde{Q}$ comme réduction et posons $F(z)=z^{p^{n}}$, de telle façon que $R$ et $Q \circ F$ aient la même réduction. Par le Lemme d'Approximation, les actions $R_{*}$ et $(Q \circ F)_{*}$ coïncident sur un voisinage de $\wp_{\text {can }}$. Comme $F_{*}$ satisfait la propriété $1, R_{*}$ satisfait aussi cette propriété.

Notons que pour tout $\delta \in \mathbb{H}_{p}$ dans un voisinage de $\delta_{\text {can }}$ on a $d\left(F_{*}(\delta), \delta_{\text {can }}\right) \geq$ $p^{n} \cdot d\left(\&, \S_{\text {can }}\right)$. Si l'on prend la fonction rationnelle $Q \in \mathbb{C}_{p}(z)$ ayant le même degré que $\widetilde{Q}$, alors $Q$ a bonne réduction et par conséquent on a $d\left(Q_{*}(\delta), \delta_{\text {can }}\right) \geq d\left(\delta, \wp_{\text {can }}\right)$ pour tout $\delta \in \mathbb{H}_{p}$, voir partie 1 de la Proposition 6.4. Le Lemme d'Approximation implique que $(Q \circ F)_{*}$ et $R_{*}$ satisfont la dernière assertion de la proposition. 


\section{Incompressiblité et preuve du Lemme Principal}

Dans ce paragraphe on termine la démonstration du Lemme Principal; elle est dans le paragraphe 11.2. Cette démonstration dépend de la Proposition 11.1, qu'on montre dans le paragraphe suivant.

11.1. Considérons pour un instant une fonction rationnelle $R$ à coefficients complexes et soit $z_{0} \in \mathbb{P}(\mathbb{C})$ un point périodique répulsif de $R$. Alors pour tout voisinage $V$ de $z_{0}$ dans $\mathbb{P}(\mathbb{C})$ la famille $\left\{\left.R^{n}\right|_{V}\right\}_{n \geq 1}$ n'est pas normale, et un théorème dû à Montel implique qu'on a

$$
\mathbb{P}(\mathbb{C}) \backslash E \subset \bigcup_{n \geq 1} R^{n}(V),
$$

où $E$ est l'ensemble exceptionnel de $R$, qui contient au plus deux points; voir par exemple [Mi].

La propriété analogue dans $\mathbb{H}_{p}$ n'est pas valable pour tout point périodique répulsif, voir l'exemple 11.2 ci-dessous. Cependant, dans la proposition suivante on montre qu'elle est vraie pour les points périodiques inséparables; ceci est à comparer avec [R3], paragraphe 5.

Proposition 11.1. Soit $R$ une fonction rationnelle à coefficients dans $\mathbb{C}_{p}$ et soit $R_{*}$ l'action induite par $R$ sur $\mathbb{H}_{p}$. Soit $\& \in \mathbb{H}_{p}$ un point périodique inséparable de $R_{*}$. Alors pour tout voisinage $\widehat{\mathcal{V}}$ de $\&$ dans $\mathbb{H}_{p}$ on a

$$
\bigcup_{n \geq 1} R_{*}^{n}(\widehat{\mathcal{V}})=\mathbb{H}_{p}
$$

Cette proposition est une conséquence simple du lemme suivant.

Étant donné un point $s \in \mathbb{H}_{p}$ et $r>0$ on pose

$$
\widehat{B}(\delta, r)=\left\{\delta^{\prime} \in \mathbb{H}_{p} \mid d\left(\delta, \delta^{\prime}\right)<r\right\} .
$$

Lemme d'Incompressibilité. Soit $R \in \mathbb{C}_{p}(z)$ une fonction rationnelle et soit $\& \in$ $\mathbb{H}_{p}$. Alors pour tout $r>0$ on a

$$
\widehat{B}\left(R_{*}(\delta), r\right) \subset R_{*}(\widehat{B}(\delta, r)) .
$$

Si de plus $\& \in \mathbb{H}_{p}$ est un point rationnel et $R_{*}$ est inséparable en $\$$, alors il existe $r_{0}>0$ tel que pour tout $r>0$,

$$
\widehat{B}\left(R_{*}(\S), r+(p-1) \cdot \min \left\{r, r_{0}\right\}\right) \subset R_{*}(\widehat{B}(\delta, r)) .
$$


Preuve. 1. Soit $\delta^{\prime} \in \mathbb{H}_{p} \backslash R_{*}(\widehat{B}(\xi, r))$. Par le Lemme 9.2, il existe $\bar{\delta} \in R_{*}^{-1}\left(\delta^{\prime}\right)$ tel que $R_{*}((\delta, \bar{\delta}))=\left(R_{*}(\delta), \varsigma^{\prime}\right)$ et tel que $R_{*}$ est injective sur $(\&, \bar{\delta})$. De plus, on a $d\left(R_{*}(\delta), \delta^{\prime}\right) \geq d(\delta, \bar{\gamma})$.

Comme $\bar{\delta} \in R_{*}^{-1}\left(\delta^{\prime}\right)$ n'appartient pas à $\widehat{B}(\delta, r)$, on a $d\left(R_{*}(\&), \delta^{\prime}\right) \geq d(\delta, \bar{\delta}) \geq r$.

2. Si $R_{*}$ est inséparable en $\delta$, alors il existe $r_{0}>0$ tel que $\operatorname{deg}_{R}(\widetilde{\delta}) \geq p>1$ pour tout $\widetilde{\delta} \in \mathbb{H}_{p}$ à distance au plus $r_{0}$ de $\delta$ (partie 1 de la Proposition 10.2).

Donc, si $\bar{\delta} \in \mathbb{H}_{p}$ est comme dans la partie 1 , on a

$$
d\left(R_{*}(\S), \digamma^{\prime}\right) \geq d(\S, \bar{\gamma})+(p-1) \cdot \min \left\{r, r_{0}\right\}
$$

(cf. Corollaire 3.7), et par conséquent $\widehat{B}\left(R_{*}(\S), r+(p-1) \cdot \min \left\{r, r_{0}\right\}\right) \subset R_{*}(\widehat{B}(\S, r))$.

Preuve de la Proposition 11.1. Soit $r>0$ tel que $\widehat{B}(\&, r) \subset \widehat{\mathcal{V}}$ et soit $r_{0}>0$ donné par le lemme précédent. On montre aisément par récurrence sur $n$ que

$$
\widehat{B}\left(\&, r+n(p-1) \min \left\{r, r_{0}\right\}\right) \subset R_{*}^{n}(\widehat{B}(\&, r)) .
$$

Exemple 11.2. Soit $c \in \mathbb{C}_{p}$ tel que $|c|=1$ et considérons la fonction rationnelle $R(z)=\frac{1}{z^{d}}+c$, où l'entier $d \geq 2$ n'est pas divisible par $p$. Elle a bonne réduction $\widetilde{R}(z)=\frac{1}{z^{d}}+\tilde{c} \in \overline{\mathbb{F}}_{p}(z)$ satisfaisant, $\widetilde{R}(0)=\infty, \widetilde{R}(\infty)=\tilde{c}$ et $\operatorname{deg}_{\widetilde{R}}(\zeta)=1$ pour $\zeta \in \overline{\mathbb{F}}_{p}-\{0\}$.

Il est facile de voir que, pour tout point $\delta \in \mathbb{H}_{p}$ tel que $\delta \prec\{|z|=1\}$ on a $d\left(R_{*}(\S), \diamond_{\text {can }}\right)=d\left(\S, \diamond_{\text {can }}\right)$. Par conséquent si $c \in \mathbb{C}_{p}$ vérifie $\widetilde{R}^{n}(\tilde{c}) \neq 0$ pour tout $n \geq 1$, alors pour tout voisinage $\widehat{\mathcal{V}}$ borné de $\delta_{\text {can }}$ dans $\mathbb{H}_{p}$, l'ensemble

$$
\bigcup_{n \geq 1} R_{*}^{n}(\widehat{\mathcal{V}})
$$

est borné. Par exemple, on peut choisir $c \in \mathbb{C}_{p}$ tel que $\widetilde{R}(\tilde{c})$ soit fixé par $\widetilde{R}$.

11.2. Démonstration du Lemme Principal. Soit $\& \in \mathbb{H}_{p}^{\mathbb{Q}}$ un point périodique inséparable de $R_{*}$ qui n'est pas exceptionnel. Quitte à remplacer $R$ par un itéré on suppose que $\&$ est fixé par $R_{*}$.

1. Soit $r>0$ suffisamment petit tel que $R_{*}$ soit inséparable en tout point rationnel contenu dans la boule $\widehat{\mathcal{V}}_{0}=\widehat{B}(\delta, r)$ et tel que pour tout $\bar{\delta}$ dans $\widehat{\mathcal{V}}_{0}$ on ait $d\left(R_{*}(\bar{\S}), \S\right) \geq p \cdot d(\bar{\S}, \S)$ (partie 2 de la Proposition 10.2).

Pour $k \geq 1$ soit $\widehat{\mathcal{V}}_{k}$ la composante connexe de $R_{*}^{-k}\left(\widehat{\mathcal{V}}_{0}\right)$ qui contient $\&$. Notons que pour chaque $k \geq 0$ l'ouvert $\widehat{\mathcal{V}}_{k+1}$ est égal à la composante connexe de $R_{*}^{-1}\left(\widehat{\mathcal{V}}_{k}\right)$ qui contient $\&$. Montrons par récurrence que pour tout $k \geq 0$ on a $\widehat{\mathcal{V}}_{k} \subset \widehat{B}\left(\delta, p^{-k} r\right)$. Cette propriété est satisfaite pour $k=0$ par définition. Supposons que l'entier $k \geq 0$ est tel 
que $\widehat{\mathcal{V}}_{k} \subset \widehat{B}\left(\delta, p^{-k} r\right)$. On a alors $\widehat{\mathcal{V}}_{k} \subset \widehat{\mathcal{V}}_{0}$ et pour tout $\bar{\delta} \in \widehat{\mathcal{V}}_{k}$ on a $d\left(R_{*}(\bar{\delta}), \S\right) \geq$ $p \cdot d(\bar{\delta}, \S)$. Comme $\widehat{\mathcal{V}}_{k} \subset \widehat{B}\left(\&, p^{-k} r\right)$ et $\widehat{\mathcal{V}}_{k+1}$ est égal à la composante connexe de $R_{*}^{-1}\left(\widehat{\mathcal{V}}_{k}\right)$ qui contient $\delta$, on a $\widehat{\mathcal{V}}_{k+1} \subset \widehat{B}\left(\delta, p^{-(k+1)} r\right)$.

Notons en particulier que la fermeture topologique de $\widehat{\mathcal{V}}_{1}$ est contenue dans $\widehat{\mathcal{V}}_{0}$ et que $\operatorname{diam}\left(\widehat{\mathcal{V}}_{k}\right) \rightarrow 0$ lorsque $k \rightarrow \infty$.

2. On montrera que pour chaque entier $k \geq 1$ l'ouvert $\widehat{\mathcal{V}}_{k}$ contient un point périodique inséparable de $R_{*}$ différent de $\$$. Ceci implique que $R_{*}$ a une infinité de points périodiques inséparables. Fixons alors un entier $k \geq 1$.

Comme $\&$ n'est pas exceptionnel, il existe un antécédent $\S^{\prime} \in \mathbb{H}_{p}$ de $\&$ par $R_{*}$, différent de 8 . Par la Proposition 11.1, on a $\bigcup_{n \geq 1} R_{*}^{n}\left(\widehat{\mathcal{V}}_{k}\right)=\mathbb{H}_{p}$. Il existe donc un point $\delta_{0} \in \widehat{\mathcal{V}}_{k}$ et un entier $n \geq 0$ tels que $R_{*}^{n}\left(\varsigma_{0}\right)=\varsigma^{\prime}$. Comme diam $\left(\widehat{\mathcal{V}}_{m}\right) \rightarrow 0$ lorsque $m \rightarrow \infty$, la partie 2 de la Proposition 10.2 implique qu'il existe un entier $m \geq 0$ tel que la composante connexe $\widehat{\mathcal{W}}$ de $R_{*}^{-(n+1)}\left(\widehat{\mathcal{V}}_{m}\right)$ qui contient $\delta_{0}$ est contenue dans $\widehat{\mathcal{V}}_{k} \backslash\{\delta\}$; voir Figure 2.

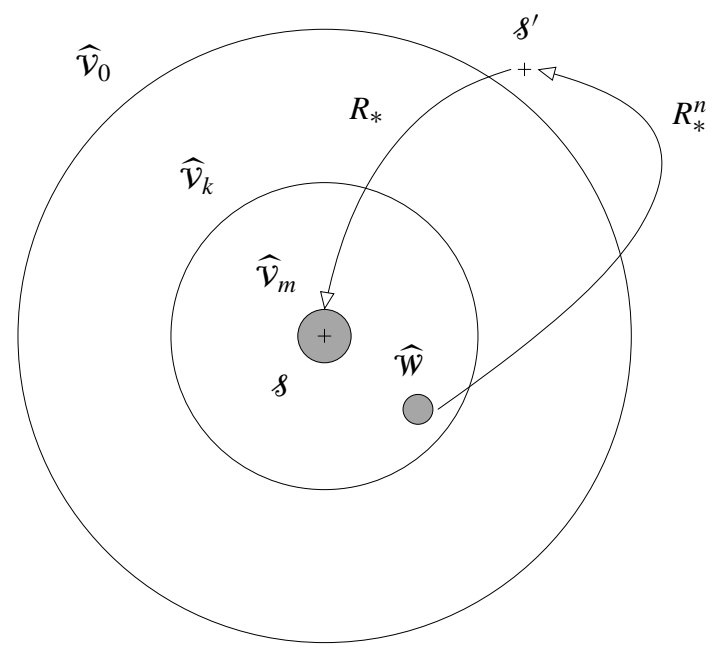

Figure 2

3. D'après la remarque qui suit l'énoncé du Lemme 9.1, les applications $R_{*}^{n+1}$ : $\widehat{\mathcal{W}} \rightarrow \widehat{\mathcal{V}}_{m}$ et $R_{*}^{m}: \widehat{\mathcal{V}}_{m} \rightarrow \widehat{\mathcal{V}}_{0}$ sont propres. L'application $R_{*}^{m+n+1}: \widehat{\mathcal{W}} \rightarrow \widehat{\mathcal{V}}_{0}$ est donc propre. Comme $\widehat{W} \subset \widehat{\mathcal{V}}_{k} \subset \widehat{\mathcal{V}}_{1}$, la fermeture topologique de $\widehat{W}$ est contenue dans $\widehat{\mathcal{V}}_{0}$ et l'ouvert $\widehat{W}$ contient un point périodique rationnel de $R_{*}$ (Corollaire 9.4). Ce point périodique est inséparable, car $R_{*}$ est inséparable en chaque point rationnel contenu dans $\widehat{\mathcal{V}}_{k} \subset \widehat{\mathcal{V}}_{0}$. De plus il est différent de $\&$, car $\widehat{\mathcal{W}} \subset \widehat{\mathcal{V}}_{k} \backslash\{\&\}$. 


\section{Appendice. Sur le nombre d'antécédents d'un point dans $\mathbb{H}_{p}$}

Le but de ce paragraphe est de montrer la proposition suivante.

Proposition. Soit $R \in \mathbb{C}_{p}(z)$ une fonction rationnelle non constante. Alors pour tout point $\& \in \mathbb{H}_{p}$ l'ensemble $R_{*}^{-1}(\&) \subset \mathbb{H}_{p}$ des antécédents de $\&$ par $R_{*}$ est fini, non vide et on $a$

$$
\sum_{R_{*}^{-1}(\delta)} \operatorname{deg}_{R}\left(f^{\prime}\right)=\operatorname{deg}(R)
$$

Ceci à été montré dans [R1] (Lemme 2.5) pour les points rationnels de $\mathbb{H}_{p}$. Ce résultat découle, dans le langage de Berkovich, du fait que $R_{*}$ est fini de degré $\operatorname{deg}(R)$ et étale au-dessus de $\mathbb{H}_{p}$; ce fait lui même est conséquence dur caractère fini, plat et génériquement étale de l'endomorphisme de la variété algébrique $\mathbb{P}_{\mathbb{C}_{p}}^{1}$ induit par $R$. Je remercie le rapporteur pour cette dernière remarque.

Fixons une fonction rationnelle $R \in \mathbb{C}_{p}(z)$. On considère d'abord le lemme suivant.

Lemme 12.1. Chaque point $s \in \mathbb{H}_{p}$ a au plus un nombre fini d'antécédents par $R_{*}$.

Preuve. Soient $\S_{1}, \ldots, \S_{k}$ des antécédents distincts de $\&$ par $R_{*}$; on montrera que $k \leq \operatorname{deg}(R)$. Choisissons un bout $\mathcal{P} \in \delta$ et pour $1 \leq i \leq k$ soit $\mathcal{P}_{i} \in \delta_{i}$ un bout tel que $R_{*}\left(\mathcal{P}_{i}\right)=\mathcal{P}$. De plus soient $\left\{C_{j}\right\}_{j \geq 0}$ et $\left\{C_{i, j}\right\}_{j \geq 0}$ chaînes évanescentes représentant $\mathcal{P}$ et $\mathcal{P}_{i}$ respectivement.

Soit $N \geq 0$ assez grand tel que les $C_{i, N}$ soient disjoints deux à deux et soit $M \geq 0$ tel que $C_{M} \subset R\left(C_{i, N}\right)$ pour $1 \leq i \leq k$. Fixons $w \in C_{M}$. Alors chaque $C_{i, N}$ contient au moins un antécédent de $w$ et par conséquent $k \leq \# R^{-1}(w) \leq \operatorname{deg}(R)$.

Preuve de la Proposition. 1. Supposons d'abord que le point $\delta \in \mathbb{H}_{p}$ ne soit pas singulier. Après changement de coordonnée on suppose $s \in(0, \infty) \subset \mathbb{H}_{p}$. Pour $r>0$ on désigne par $\oint_{r} \in(0, \infty)$ le point de $\mathbb{H}_{p}$ associé à la boule $\{|z|<r\}$.

1.1. Soit $X \subset \mathbb{P}\left(\mathbb{C}_{p}\right)$ l'ensemble des zéros et des pôles de $R$ et soit $\widehat{X} \subset \mathbb{H}_{p}$ l'enveloppe convexe de $X$. Comme $X$ a au moins deux éléments, l'ensemble $\widehat{X}$ est non vide.

Soit $\varsigma^{\prime} \in \mathbb{H}_{p}$ un point tel que $R_{*}\left(\mathcal{S}^{\prime}\right) \in(0, \infty)$. Alors il existe des bouts $\mathcal{P}_{0}, \mathcal{P}_{\infty} \in$ $\delta^{\prime}$ tel que $0 \in B_{R_{*}\left(\mathcal{P}_{0}\right)}$ et $\infty \in B_{R_{*}\left(\mathcal{P}_{\infty}\right)}$. Par le Lemme 3.2, les boules $B_{\mathcal{P}_{0}}$ et $B_{\mathcal{P}_{\infty}}$ intersectent $X$. Le point $\delta^{\prime}$ appartient donc à $\bar{X}$ et par conséquent $R_{*}^{-1}((0, \infty)) \subset \widehat{X}$.

1.2. Considérons la fonction

$$
r \mapsto \sum_{\delta^{\prime} \in R_{*}^{-1}\left(s_{r}\right)} \operatorname{deg}_{R}\left(\delta^{\prime}\right)
$$


Elle prend la valeur $\operatorname{deg}(R)$ pour $r>0$ petit (cf. Proposition 3.4 et (3)); il suffit alors de montrer qu'elle est localement constante.

Fixons $r_{0}>0$ et soit $\mathcal{P}_{0} \in s_{r_{0}}$ le bout tel que $0 \in B_{\mathcal{P}_{0}}$. Pour chaque $\delta^{\prime} \in$ $R_{*}^{-1}\left(\wp_{r_{0}}\right)$ et chaque $\mathcal{P}^{\prime} \in \mathcal{S}^{\prime}$ tel que $R_{*}\left(\mathcal{P}^{\prime}\right)=\mathcal{P}_{0}$, soit $\bar{\delta}\left(\mathcal{P}^{\prime}\right) \in \mathbb{H}_{p}$ un point donné par la Proposition 3.5, de telle façon que $R_{*}$ soit injective sur $\left(\mathscr{S}^{\prime}, \bar{\delta}\left(\mathcal{P}^{\prime}\right)\right.$ ] et tel qu'on ait $\operatorname{deg}_{R}(\widehat{\S})=\operatorname{deg}_{R}\left(\mathcal{P}^{\prime}\right)$ pour $\widehat{\S} \in\left(\mathcal{S}^{\prime}, \bar{\delta}\left(\mathcal{P}^{\prime}\right)\right]$. On peut supposer que $R_{*}\left(\left[\mathcal{S}^{\prime}, \bar{\delta}\left(\mathcal{P}^{\prime}\right)\right]\right) \subset(0, \infty)$ et que $\bar{\delta}\left(\mathcal{P}^{\prime}\right) \in \widehat{X}$. On a donc $\left[\mathcal{S}^{\prime}, \bar{\gamma}\left(\mathcal{P}^{\prime}\right)\right] \subset \bar{X}$.

Soit $r \in\left(0, r_{0}\right)$ proche de $r_{0}$. Alors chacun des segments $\left(\mathcal{S}^{\prime}, \bar{\delta}\left(\mathcal{P}^{\prime}\right)\right]$ contient exactement un antécédent de $\delta_{r}$ par $R_{*}$.

Considérons d'autre part $\tilde{\delta} \in R_{*}^{-1}\left(\delta_{r}\right)$; par la partie 1.1 on a $\tilde{\&} \in \widehat{X}$. Alors il existe $\delta^{\prime} \in R_{*}^{-1}\left(f_{r_{0}}\right)$ à distance au plus $r_{0}-r$ de $\widetilde{\delta}$ (Lemme 9.2). Si l'on désigne par $\mathcal{P}^{\prime}$ le bout dans $\S^{\prime}$ tel que $\widetilde{\delta} \prec B_{\mathcal{P}^{\prime}}$, alors on a $\tilde{\S} \in\left(\mathcal{S}^{\prime}, \bar{\gamma}\left(\mathcal{P}^{\prime}\right)\right]$, lorsque $r$ est suffisamment proche de $r_{0}$, voir Lemme 2.9. En particulier, $\operatorname{deg}_{R}(\widetilde{\mathscr{S}})=\operatorname{deg}_{R}\left(\mathcal{P}^{\prime}\right)$.

On a alors,

$$
\sum_{\tilde{\wp} \in R_{*}^{-1}\left(\delta_{r}\right)} \operatorname{deg}_{R}(\tilde{\wp})=\sum_{\delta^{\prime} \in R_{*}^{-1}\left(\delta_{r_{0}}\right)} \sum_{\mathcal{P}^{\prime} \in \mathcal{S}^{\prime}, R_{*}\left(\mathcal{P}^{\prime}\right)=\mathcal{P}_{0}} \operatorname{deg}_{R}\left(\mathcal{P}^{\prime}\right) .
$$

Lorsque $r_{0} \in\left|\mathbb{C}_{p}^{*}\right|$ chaque point $\delta^{\prime} \in R_{*}^{-1}\left(\delta_{r_{0}}\right)$ est rationnel et la Proposition 3.3 implique qu'on a

$$
\sum_{\mathcal{P}^{\prime} \in \mathcal{S}^{\prime}, R_{*}\left(\mathcal{P}^{\prime}\right)=\mathcal{P}_{0}} \operatorname{deg}_{R}\left(\mathcal{P}^{\prime}\right)=\operatorname{deg}_{R}\left(\mathcal{S}^{\prime}\right) .
$$

Lorsque $r_{0} \notin\left|\mathbb{C}_{p}^{*}\right|$ chaque point $\delta^{\prime}$ est irrationnel et contient un unique bout $\mathcal{P}^{\prime}$ tel que $R_{*}\left(\mathcal{P}^{\prime}\right)=\mathscr{P}_{0}$. De plus $\operatorname{deg}_{R}\left(\mathcal{P}^{\prime}\right)=\operatorname{deg}_{R}\left(\mathcal{S}^{\prime}\right)$ et donc l'égalité précédente est aussi valable dans ce cas. Dans tous les cas on a donc

$$
\sum_{\tilde{s} \in R_{*}^{-1}\left(\delta_{r}\right)} \operatorname{deg}_{R}(\tilde{\delta})=\sum_{\delta^{\prime} \in R_{*}^{-1}\left(\delta_{r_{0}}\right)} \operatorname{deg}_{R}\left(\xi^{\prime}\right) .
$$

Le cas $r>r_{0}$ proche de $r_{0}$ est analogue.

2. Supposons maintenant $\delta=\{\mathcal{P}\} \in \mathbb{H}_{p}$ singulier. Pour tout $i$ compris entre 1 et $k$ notons $\delta_{i}=\left\{\mathcal{P}_{i}\right\} \in \mathbb{H}_{p}$ les antécédents de $\&$ par $R_{*}$. Pour chaque $1 \leq i \leq k$ soit $\bar{\delta}_{i} \in \mathbb{H}_{p}$ donné par la Proposition 3.5, de telle façon que $R_{*}$ soit injective sur

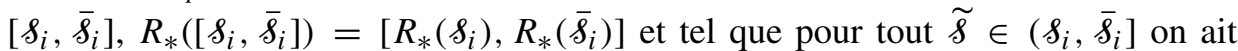
$\operatorname{deg}_{R}(\widetilde{\delta})=\operatorname{deg}_{R}\left(\mathcal{P}_{i}\right)=\operatorname{deg}_{R}\left(\delta_{i}\right)$.

Quitte à prendre les points $\bar{\delta}_{i}$ plus proches des $\delta_{i}$, on suppose que les segments $\left[\delta_{i}, \bar{\delta}_{i}\right]$ sont deux à deux disjoints et que $\bar{\delta}=R_{*}\left(\bar{\gamma}_{i}\right)$ est un point non singulier qui ne dépend pas de $1 \leq i \leq k$.

2.1. Supposons par l'absurde qu'il existe un antécédent $\widehat{\S}$ de $\bar{\delta}$ distinct des $\bar{\delta}_{i}$. Notons alors que pour chaque $1 \leq i \leq k$ le point $\widehat{\delta}$ n'appartient à $\left[\wp_{i}, \bar{\delta}_{i}\right]$. Le 
Lemme 9.2 implique qu'il existe $1 \leq i \leq k$ tel que $R_{*}$ soit injective sur [ $\S_{i}, \widehat{\jmath}$ ]. Notons alors que $\bar{\delta}_{i}$ n'appartient pas à $\left[\delta_{i}, \widehat{\jmath}\right]$. De plus, le point $\delta_{i}$ n'est pas entre $\widehat{\jmath}$ et $\bar{\delta}_{i}$ car il est singulier. Le Lemme 2.8 implique alors qu'il existe un point rationnel $\delta^{\prime}$ qui est entre $\delta_{i}$ et $\bar{\delta}_{i}$, entre $\bar{\gamma}_{i} \bar{\gamma}_{i}$ et $\widehat{\S}$ et entre $\widehat{\gamma}$ et $\delta_{i}$.

Soient $\overline{\mathcal{P}}, \widehat{\mathcal{P}} \in \delta^{\prime}$ les bouts tels que $\bar{\delta}_{i} \prec B_{\overline{\mathcal{P}}}$ et $\widehat{\mathcal{S}} \prec B_{\widehat{\mathcal{P}}}$. Comme pour tout point $\widetilde{\S}$ dans $\left(\wp_{i}, \bar{\wp}_{i}\right)$ on a $\operatorname{deg}_{R}(\widetilde{\S})=\operatorname{deg}_{R}\left(\wp_{i}\right)=\operatorname{deg}_{R}\left(\wp^{\prime}\right)$, on conclut que $\operatorname{deg}_{R}(\overline{\mathcal{P}})=\operatorname{deg}_{R}\left(\delta^{\prime}\right)$ (cf. Proposition 3.5). On a donc $R_{*}(\widehat{\mathcal{P}}) \neq R_{*}(\overline{\mathcal{P}})$ (cf. partie 3 de la Proposition 3.3). Comme $R_{*}$ est injective sur $\left[\wp_{i}, \bar{\wp}_{i}\right]$ (resp. $\left[\wp_{i}, \widehat{\S}\right]$ ) on a $\bar{\delta}=R_{*}\left(\bar{g}_{i}\right) \prec B_{R_{*}(\overline{\mathcal{P}})}\left(\right.$ resp. $\left.\bar{\S}=R_{*}(\widehat{\jmath}) \prec B_{R_{*}(\widehat{\mathcal{P}})}\right)$. Comme les bouts $R_{*}(\overline{\mathcal{P}})$ et $R_{*}(\widehat{\mathcal{P}})$ sont distincts et appartiennent au même point, les boules correspondantes sont disjointes et on obtient une contradiction.

2.2. Par la partie 1 on a

$$
\begin{aligned}
\sum_{\tilde{\zeta} \in R_{*}^{-1}(\S)} \operatorname{deg}_{R}(\tilde{\S}) & =\sum_{1 \leq i \leq k} \operatorname{deg}_{R}\left(\delta_{i}\right)=\sum_{1 \leq i \leq k} \operatorname{deg}_{R}\left(\bar{\gamma}_{i}\right) \\
& =\sum_{\delta^{\prime \prime} \in R_{*}^{-1}(\bar{\gamma})} \operatorname{deg}_{R}\left(\delta^{\prime \prime}\right)=\operatorname{deg}(R) .
\end{aligned}
$$

\section{Références}

[Ben1] R. Benedetto, Hyperbolic maps in p-adic dynamics. Ergodic Theory Dynam. Systems 21 (2001), 1-11. Zbl 0972.37027 MR 1826658

[Ben2] R. Benedetto, Reduction, dynamics, and Julia sets of rational functions. J. Number Theory 86 (2001), 175-195. Zbl 0978.37039 MR 1813109

[Bez] J. P. Bézivin, Sur la compacité des ensembles de Julia des polynômes $p$-adiques. Math. Z. 246 (2004), 273-289. Zbl 1047.37031 MR 2031456

[BGR] S. Bosch, U. Güntzer, R. Remmert, Non-Archimedean analysis. A systematic approach to rigid analytic geometry. Grundlehren Math. Wiss. 261, Springer-Verlag, Berlin 1984. Zbl 0539.14017 MR 07469615

[Ha] R. Hartshorne, Algebraic geometry. Grad. Texts in Math. 52, Springer-Verlag, New York, Heidelberg, Berlin 1977. Zbl 0367.14001 MR 0463157

[Hs] L. C. Hsia, Closure of periodic points over a non-Archimidean field. J. London Math. Soc. (2) 62 (2000), 685-700. Zbl 1022.11060 MR 1794277

[Mi] J. Milnor, Dynamics in one complex variable. Vieweg, Wiesbaden 1999. Zbl 0946.30013 MR 1721240

[MS] P. Morton, J. Silverman, Periodic points, multiplicities, and dynamical units. J. Reine Angew. Math. 461 (1995), 81-122. Zbl 0813.11059 MR 13242105

[R1] J. Rivera-Letelier, Dynamique des fonctions rationnelles sur des corps locaux. Astérisque 287 (2003), 147-230. Zbl 02066305 MR 2040006 
Vol. 80 (2005)

[R2] J. Rivera-Letelier, Espace hyperbolique $p$-adique et dynamique des fonctions rationnelles. Compositio Math. 138 (2003), 199-231. Zbl 1041.37021 MR 2018827

[R3] J. Rivera-Letelier, Sur la structure des ensembles de Fatou $p$-adiques. Prépublication 2002, Arxiv math. DS/0412180.

Received September 20, 2003; revised September 11, 2004

Juan Rivera-Letelier, Departamento de Matemáticas, Universidad Católica del Norte, Casilla

1280, Antofagasta, Chile

E-mail: juanrive@ucn.cl 